\title{
Universality of dissipative self-assembly from quantum dots to human cells
}

\author{
Ghaith Makey ${ }^{\circledR 1,2}$, Sezin Galioglu ${ }^{1}{ }^{1}$, Roujin Ghaffari', E. Doruk Engin ${ }^{1}{ }^{3}$, Gökhan Yıldırım $\mathbb{D}^{4}$, \\ Özgün Yavuz $\mathbb{1}^{5}$, Onurcan Bektaș $\mathbb{1}^{6,7}$, Ü. Seleme Nizam ${ }^{8}{ }^{8}$, Özge Akbulut $\mathbb{D}^{9}$, Özgür Șahin $\left(\mathbb{D}^{9,10}\right.$, \\ Kıvanç Güngör (1) ', Didem Dede ${ }^{1}{ }^{1}$, H. Volkan Demir ${ }^{1,2,5,11}$, F. Ömer Ilday $\mathbb{D}^{1,2,5}$ and Serim Ilday $\mathbb{1}^{1 凶}$
}

\begin{abstract}
An important goal of self-assembly research is to develop a general methodology applicable to almost any material, from the smallest to the largest scales, whereby qualitatively identical results are obtained independently of initial conditions, size, shape and function of the constituents. Here, we introduce a dissipative self-assembly methodology demonstrated on a diverse spectrum of materials, from simple, passive, identical quantum dots (a few hundred atoms) that experience extreme Brownian motion, to complex, active, non-identical human cells $\left(-10^{17}\right.$ atoms $)$ with sophisticated internal dynamics. Autocatalytic growth curves of the self-assembled aggregates are shown to scale identically, and interface fluctuations of growing aggregates obey the universal Tracy-Widom law. Example applications for nanoscience and biotechnology are further provided.
\end{abstract}

Tis: he idea of self-assembly research is to design and build planned structures and functionalities, starting from simple building blocks. A vast body of work points to the possibility and effectiveness of this approach. However, two major challenges remain: incorporation of dynamic adaptive characteristics into self-assembly methodologies, and unification of self-assembly methodologies that transcend the specificity of the systems that are being studied.

Progress towards addressing the first challenge is exciting and promising $^{1,2}$ : studies on hydrogels ${ }^{3,4}$, polymers ${ }^{5,6}$, microtubules $^{7,8}$, oscillatory chemical reactions $s^{2,9}$ and colloids ${ }^{10-13}$ have shown that self-assembled and organized systems can respond to the changes in their environments when driven out of equilibrium. Recently, we reported dynamic adaptive colloidal crystals (formed by tens to thousands of particles) of a multiplicity of patterns (hexagonal, square, oblique lattices and Moiré patterns) in a system that operates far from equilibrium under highly nonlinear and strongly stochastic conditions ${ }^{14}$. Despite all these studies, a thorough understanding of the underlying principles and a theoretical framework to describe such dynamic adaptive phenomena in non-equilibrium systems is lacking ${ }^{15-18}$.

The second challenge is intricately coupled to the first. However, it has only been approached from the perspective of computer algorithms (for example, cellular automata, tile assembly models) ${ }^{19,20}$ and diffusion- or reaction-limited fractal aggregates of particles ${ }^{21,22}$. Developing a universal self-assembly methodology that is applicable to almost any material, whereby qualitatively identical results are obtained independently of initial conditions, size, shape and function of the constituents, remains unresolved.

Here, we address the second challenge by presenting a universal dissipative self-assembly methodology with which we can assemble, disassemble and control movement of living and non-living, simple and complex, passive and active, identical and non-identical particles with substantial implications for the first challenge. We show that their autocatalytic growth follows a single sigmoidal curve even when the entities being self-assembled differ in size by more than three orders of magnitude. Interface fluctuations of growing aggregates follow the universal Tracy-Widom distribution arising from various many-body systems with interacting and correlated constituents $^{23-33}$. Further, we demonstrate that the aggregates can take arbitrary geometrical forms, and these forms can actively be moved, transported and rotated. Proof-of-principle demonstrations for potential applications in nanoscience and biotechnology are also provided.

\section{Mechanism}

The physical mechanism is simple in that it involves a quasi-twodimensional (2D) confined material of interest suspended in a liquid, subject mainly to two physical forces: fluid flow (induced by the spatiotemporal thermal gradients created via a femtosecond laser) and Brownian motion. The effects of various other forces that may be present in the different material systems that we investigate here (that is, phoretic forces, chemical gradients in growth media of living organisms, electrostatic interactions) are relatively weak compared with the two dominant physical forces. This simplicity is essential to liberate the self-assembly process from microscopic details of the system, and thereby to achieve universality.

Schematic representations in Fig. 1a,b depict the experimental settings and the range of particles and living organisms that are used in this study. The solutions of particles and organisms are quasi-2D confined between two thin glass slides, and the energy flux is supplied through an ultrafast laser to drive the system far

'UNAM-National Nanotechnology Research Center and Institute of Materials Science and Nanotechnology, Bilkent University, Ankara, Turkey. ${ }^{2}$ Department of Physics, Bilkent University, Ankara, Turkey. ${ }^{3}$ Institute of Biotechnology, Ankara University, Ankara, Turkey. ${ }^{4}$ Department of Mathematics, Bilkent University, Ankara, Turkey. ${ }^{5}$ Department of Electrical and Electronics Engineering, Bilkent University, Ankara, Turkey. ${ }^{6}$ Department of Physics, Middle East Technical University, Ankara, Turkey. ${ }^{7}$ Department of Mathematics, Middle East Technical University, Ankara, Turkey. ${ }^{8}$ Department of Physics, Boğaziçi University, İstanbul, Turkey. ${ }^{9}$ Department of Molecular Biology and Genetics, Bilkent University, Ankara, Turkey. ${ }^{10}$ Department of Drug Discovery and Biomedical Sciences, University of South Carolina, Columbia, SC, USA. "LUMINOUS! Center of Excellence for Semiconductor Lighting and Displays, School of Electrical and Electronic Engineering, School of Physical and Mathematical Sciences, Nanyang Technological University, Singapore, Singapore. 凶e-mail: serim@bilkent.edu.tr 


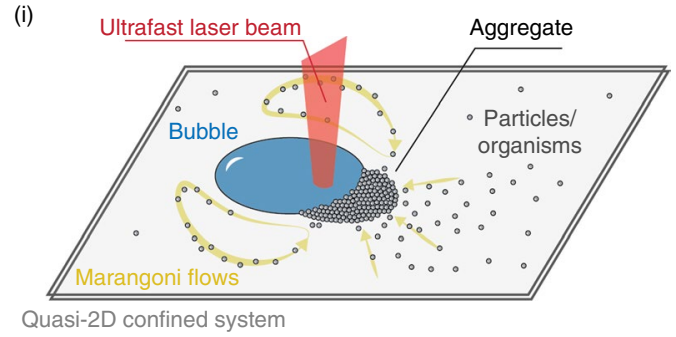

(ii)

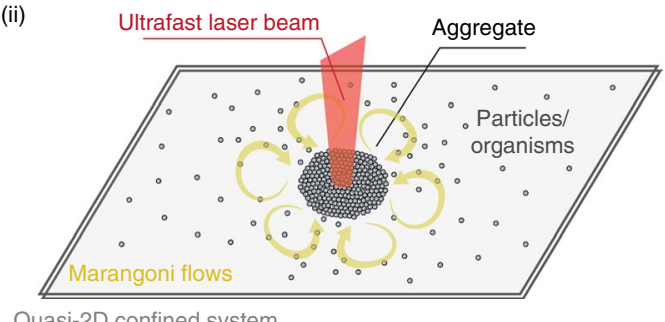

b

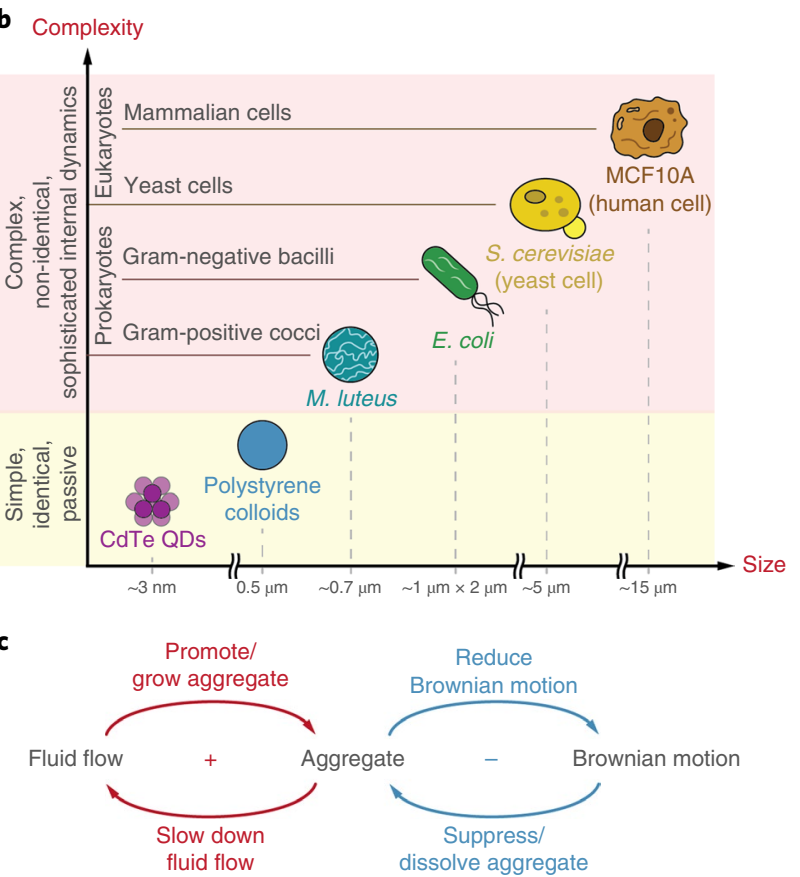

Fig. 1 | Universal dissipative self-assembly methodology. a-c, Aggregation of particles and organisms adjacent to a cavitation bubble (i) or at the laser spot (ii) in a quasi-2D setting through ultrafast laser-induced flows (a), complexity and size distribution of the particles and organisms used in this study (b) and intrinsic positive and negative feedback mechanisms that control the dynamics of the self-assembly process (c).

from equilibrium. The laser wavelength of $1,040 \mathrm{~nm}$ ensures that optical absorption is predominantly nonlinear, which arises from multiphoton absorption of ultrafast pulses in glass and liquid. This creates extremely steep, well-controlled spatiotemporal thermal gradients that may instantaneously reach peak values of $\sim 10^{6} \mathrm{~K} \mathrm{~mm}^{-1}$, despite $<0.11 \mathrm{~mW}$ of average absorption, which is essential for not overheating the liquid (see Supplementary Information for details). Thermal gradients, together with surface forces, induce Marangoni flows (Extended Data Fig. 1), which drag the material of interest towards aggregation, either adjacent to a cavitation bubble, created when the optical breakdown threshold is reached ${ }^{34}$, or at the beam spot (Supplementary Video 1).

Particles or organisms forming an aggregate interact with each other through the fluid. Each entity feels the drag force of the fluid if it is flowing, and of course, the Brownian forces. Because the thickness of the liquid film is comparable to the size of the entities, each particle or organism influences the flow of fluid around itself. For a single, isolated particle or organism, this effect is quite small and does not have a substantial influence over distances of a few micrometres. However, once a critical number of entities accumulates in a given area, forming a seed aggregate, they start acting as a sieve (due to the voids between neighbouring particles or organisms), which, in turn, slows down fluid flow around the aggregate ${ }^{14}$. Newly arriving dragged particles or organisms also slow down with the flow, and therefore join the aggregate upon contact rather than scatter from it. This relation between the fluid flow and the aggregate forms a positive feedback loop (Fig. 1c).

There is also a negative feedback loop between the aggregate and the Brownian force (Fig. 1c). The aggregate reduces Brownian motion of the entities that join it, and in return Brownian motion suppresses further growth of the aggregate if its magnitude is comparable to that of the drag force. In the absence of laser light, Brownian motion dissolves the aggregate and distributes the entities back into the system homogeneously.

These positive and negative feedback loops form an intrinsic feedback mechanism (Fig. 1c) that controls this otherwise uncontrollable highly nonlinear (coupling of fluid flow with particle positions and bubble dynamics) and strongly stochastic (resulting from the Brownian motion) system. This follows the well known slaving principle of ref. ${ }^{35}$. When nearly all degrees of freedom become mutually locked through nonlinear feedback mechanisms, astronomically many degrees of freedom can be controlled using just a handful of control 'knobs'. We use only the laser power and the beam position.

The laser power determines the magnitude of the drag force. Increasing the laser power quickens fluid flow, which carries more particles to the aggregate within a shorter time. The aggregate, then, grows to form quite large crystals, even with thousands of units, as we reported earlier ${ }^{14}$. The reason is that negative feedback is suppressed in the presence of dominant positive feedback, so the aggregate tends to grow as long as the system is not depleted of particles. If the laser power is decreased to a level at which the magnitude of the drag force is comparable to that of the Brownian force, the system reaches a steady state under sustained far-fromequilibrium conditions. As a result, the overall size, position and even configuration of the aggregates are stable.

The beam position controls the distribution of spatiotemporal thermal gradients and physical boundaries in the system: sensitivity to the beam position is due to nonlinear absorption of ultrafast pulses. Multiphoton absorption ensures that thermal gradients can be altered when the beam is moved from one spatial position to another at the relevant temporal scale (here, within a few seconds). New bubbles of any size, geometry and distance can be created at the new beam position, which introduces new physical boundaries that can alter the overall dynamics of the self-assembly process. None of these is trivial with linear absorption of laser pulses.

\section{Results}

Experimental demonstration. Universal dissipative self-assembly is performed on (i) 3-nm CdTe quantum dots, (ii) $500-\mathrm{nm}$ pure polystyrene spheres, (iii) $\sim 0.7-\mu \mathrm{m}$ soft spheres of non-motile Micrococcus luteus bacterial cells, (iv) $\sim 1-\mu \mathrm{m} \times 2-\mu \mathrm{m}$ rod-like, 


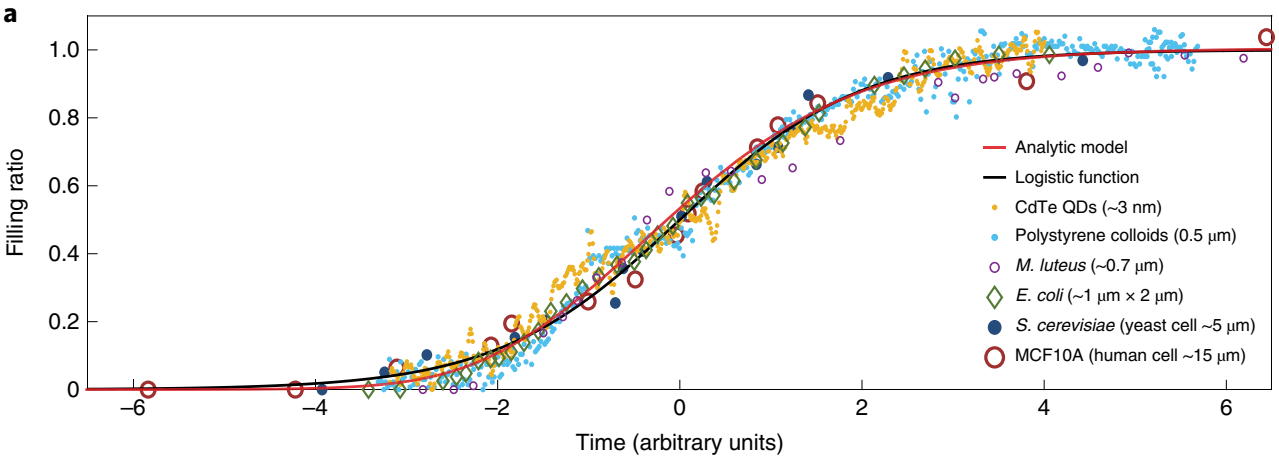

b
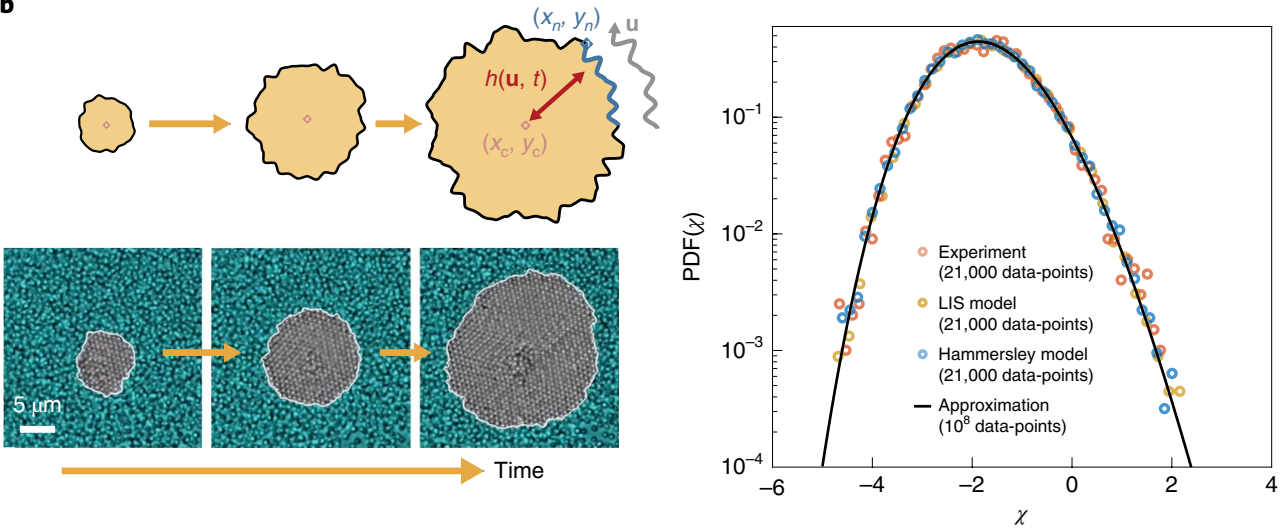

Fig. 2 | Universal dynamics. a, Graph showing the filling ratio of a selected area with particles and organisms during their aggregation. Growth curves of all entities are shown to collapse into a single sigmoidal curve. b, Illustration (top) and time-lapse microscopy images (bottom) showing circularly growing aggregates, using a $\times 100$ objective for the imaging. Height distributions of growing interfaces were calculated along the aggregate boundary for each pixel of all frames, where $\mathbf{u}$ is the vector connecting a given point along the boundary to the centroid. The semilog-scale plot shows the probability distribution function (PDF) of the average interface fluctuations for experiments (red circles), the Hammersley (blue circles) and LIS (yellow circles) models and the analytic approximation (solid line) following the Tracy-Widom GUE distribution.

motile Escherichia coli bacterial cells, (v) $\sim 5-\mu \mathrm{m}$ elliptical, nonmotile Saccharomyces cerevisiae yeast cells and (vi) $\sim 15-\mu \mathrm{m}$ nonmotile (in suspension) MCF10A normal human breast cells. As shown in Fig. 1b, all these particles and organisms are completely different in size, mass, geometry and internal dynamics.

We start with few-nanometre quantum dots comprising only a few hundred atoms each and polystyrene colloids that are more than two orders of magnitude larger than the quantum dots. Particles of each type are simple, passive, identical and subject to strong fluctuations due to Brownian motion being extremely powerful, given their tiny masses.

Next, we switch to living organisms, which are complex, active and adaptive with sophisticated internal dynamics. Cells of $M$. luteus (a prokaryote organism) are similar to colloids in size and geometry, but their boundaries are soft and elastic with a rough texture. E. coli (also a prokaryote organism) is surrounded by numerous fimbria to adhere and a flagellum to direct its motion. Cells of $S$. cerevisiae, a model organism for eukaryotes, have even more complex morphological arrangements such as inner compartments including a nucleus, mitochondrion and ribosomes along with a cell membrane. Finally, we experimented on human cells, the most complex of all the constituents considered here, where each cell is comprised of some $10^{17}$ atoms, which interact through sophisticated internal dynamics.

The liquid media of all particles and organisms also have necessarily completely different compositions: quantum dots and colloids are suspended in water, living organisms in specific growth media (Methods).
Regardless of all these critical differences, the assembly and disassembly dynamics of all six systems are qualitatively the same: particles and organisms are dragged by the laser-driven fluid flows towards their aggregation at bubble boundaries when the laser is turned on. When the laser is turned off, fluid flows are no longer active; as a result, aggregates dissolve due to Brownian motion, as demonstrated in Supplementary Video 2 and Extended Data Fig. 2. Dissolution of the aggregates for small and large entities is quantitatively different because the magnitude of the Brownian force scales down with increasing mass. It is $>1,000$ times stronger for the quantum dots $(\sim 3 \mathrm{~nm})$ than for the human cells $(\sim 15 \mu \mathrm{m})$.

Scaling of the autocatalytic growth. Previously, we showed that the aggregation of polystyrene spheres was autocatalytic, following a sigmoidal curve that matches the logistic function and our analytical toy model ${ }^{14}$. Here, we further show that the aggregations of quantum dots and living organisms are also autocatalytic and universal, following the same sigmoidal curve (Fig. 2a), apart from naturally having different timescales (Methods and Extended Data Figs. 3 and 4).

Scaling of the autocatalytic growth is expected because the aggregation dynamics depends only on the intrinsic feedback mechanisms between the fluid flow, aggregate and Brownian motion. The collective sieve effect of the entities decreases the velocity of fluid flow in the vicinity of an aggregate (Darcy's law ${ }^{14,36}$ ), which, in turn, significantly increases the probability of entities joining the aggregate, thereby further growing it. A larger aggregate acts as a larger sieve, thereby reducing flow speed over a larger area, which 

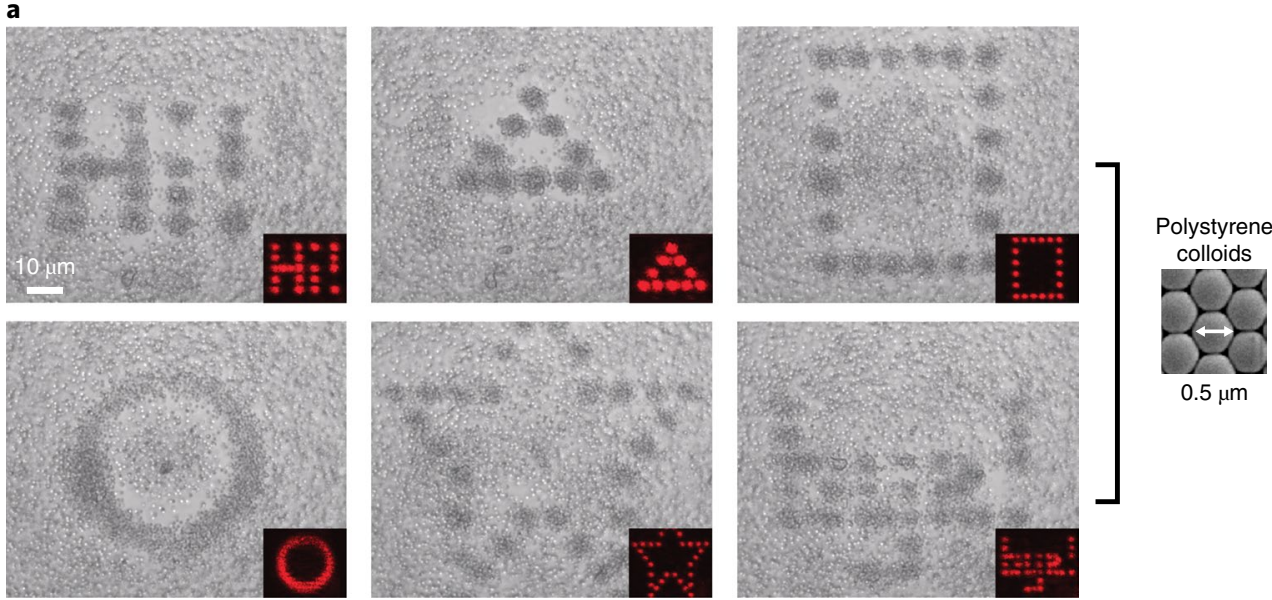

b
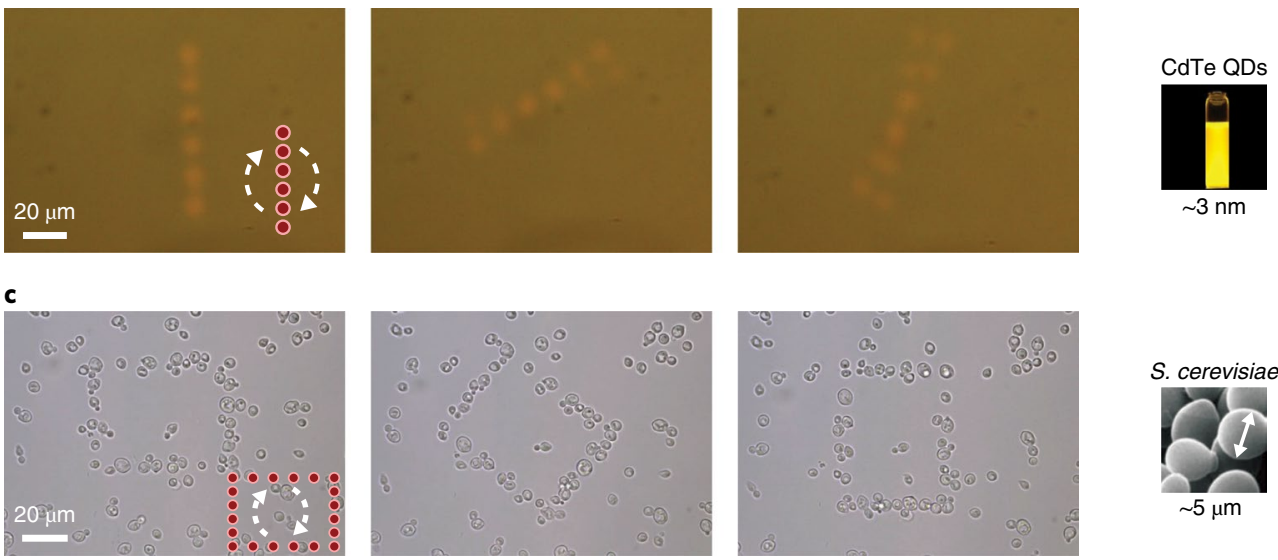

Fig. 3 | Spatiotemporal control over the aggregates. a-c, Time-lapse microscopy images showing that aggregates of 0.5- $\mu$ m polystyrene spheres can be patterned to make words and geometrical shapes following the beam patterns (insets), using a $\times 40$ objective (a), and that aggregates of $\sim 3 \mathrm{~nm}$ quantum dots (QDs) (b) and $\sim 5-\mu \mathrm{m}$ S. cerevisiae yeast cells (c) can be patterned and rotated following the beam patterns and rotations shown in the inset images, using a $\times 40$ objective.

recruits more entities to the aggregate. Eventually, growth saturates due to the finite extent over which flows are induced by the laser and also because the entities are depleted from the vicinity of the aggregates. These mechanisms result in a sigmoidal growth curve, which is commonly observed in diverse complex, dynamic adaptive systems ${ }^{37-39}$.

Scaling of the fluctuations. The experimental demonstration and scaling of the autocatalytic growth curve are related to the average number of entities participating in the aggregation process. We also find that the deviations from the average, more specifically fluctuations of the growing interfaces of the aggregates, also scale universally. In particular, we show that statistics of the probability distribution of fluctuations are consistent with those of the TracyWidom distribution ${ }^{23,24}$. This asymmetric and non-Gaussian distribution is known to be universal, arising in various many-body systems with interacting and correlated constituents such as random matrices, stochastic surface growth, directed polymers, traffic flow, random tilings, stock prices and so on ${ }^{25-33}$.

To collect enough data for reliable statistics, we performed Tracy-Widom analyses on polystyrene spheres. The analyses were performed on circularly growing aggregates (Fig. $2 \mathrm{~b}$ and Supplementary Video 3) for a Tracy-Widom Gaussian unitary ensemble (GUE) distribution that belongs to the Kardar-ParisiZhang universality class ${ }^{22,28,40}$. To detect the boundary (perimeter) of the growing aggregates in a highly dense and strongly fluctuating environment, we developed an interface tracking algorithm, with which the boundaries of approximately 21,000 frames from 14 different experiments were detected (Methods).

We compared our experimental findings with two different systems that are known to generate a Tracy-Widom GUE distribution, namely, Hammersley's interacting-particle process ${ }^{41}$ and the length statistic of the longest increasing subsequence (LIS) of a uniform random permutation from combinatorics ${ }^{42-44}$. As with any statistical distribution, the idealized results are expected to be achieved asymptotically for infinitely many samples, and one expects to observe gradual convergence for increasing sample size. For the Hammersley and LIS models, we deliberately used a sample size equivalent to that of our experiments to provide a realistic benchmark for how close an agreement with the ideal case we should expect, given our finite sample size. For the analytic approximation $^{42}$, we used a sample size of $10^{8}$ (Methods).

The semilogarithmic plot in Fig. $2 \mathrm{~b}$ presents a comparison of the probability distribution of the temporal roughness of mean height per aggregate over time (red circles) with the curve obtained by Hammersley (blue circles) and LIS (yellow circles) models and the numerical data of the analytic approximation ${ }^{42}$ (solid line). The plot shows that all four probability distribution functions are in good agreement. To quantify how closely statistical distributions of the four match each other, we calculated and compared all the 
a

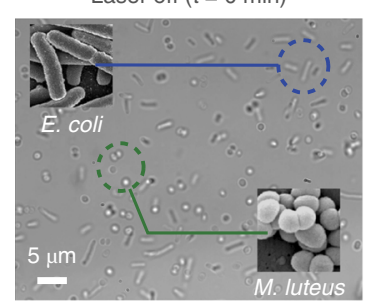

Laser on ( $t=1 \mathrm{~min})$

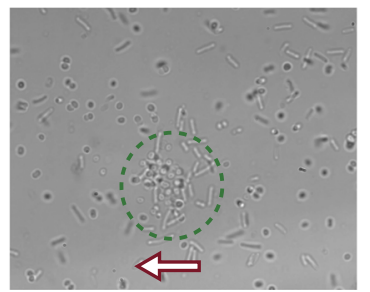

Laser on ( $t=2 \mathrm{~min})$

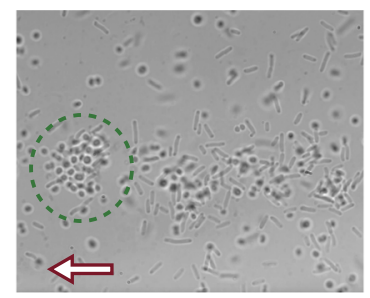

Laser on $(t=3 \mathrm{~min})$

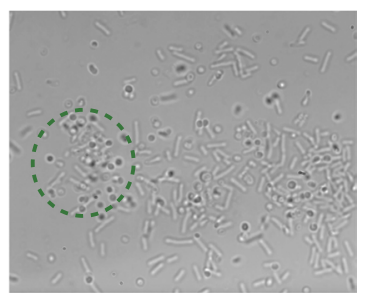

Stop moving the laser beam $(t=6 \mathrm{~s})$

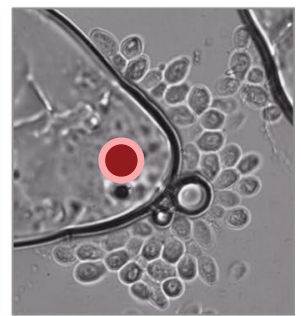

Fig. 4 | Proof-of-principle demonstrations on living organisms. a,b, Time-lapse microscopy images showing separation of $M$. luteus (gram-positive) and E. coli (gram-negative) bacterial cells from an initially homogeneous mixture, using a $\times 100$ objective (a), and formation of vertex flows, which stirs $S$. cerevisiae yeast cells when the laser beam is moved from right to left, using a $\times 40$ objective (b).

moments up to the eighth normalized moment (Supplementary Table 1). These results indicate that the experimentally measured fluctuations exhibit as much agreement with an idealized TracyWidom GUE distribution as one would expect, given the sample size of 21,000 .

In our previous study, we showed that when the laser is off the fluctuations are independent of the number of particles in a given area (uncorrelated random variables), obeying the central limit theorem. However, this result does not apply when the laser is turned on because the fluctuations are not independent anymore. Instead, the system exhibits giant number fluctuations ${ }^{14}$ with an asymmetric and non-Gaussian distribution. Now, we know that the distribution of the fluctuations obeys the Tracy-Widom law, and they depend on the number of particles in a given area (correlated variables). Next, we investigated if the cause of these correlations is the thermal-gradient-induced flows or if it is the feedback that correlates the positions of the particles, which manifests even in their fluctuations.

To answer this question, we performed a control experiment, where we created directional flow in the absence of the laser light: we prepared the samples as we did for the Tracy-Widom analysis, only this time we kept one end of the samples open to the ambient atmosphere (Extended Data Fig. 5). Evaporation of the fluid from the open end created a unidirectional flow due to the pressure difference between the sample and the room, which dragged Brownian particles towards the open end of the sample (Supplementary Video 4). Next, we defined a virtual boundary in the form of a line perpendicular to the flow. This boundary is used to stand for the physical boundary that the particles were supposed to hit and collect to. To create the virtual aggregate, we detected and traced the centroids of each colloid and their landing positions. Experimental boundaries of approximately 90,000 frames were detected from these control experiments to perform the statistical analysis (Methods).

The result of the control experiments shows that the aggregation is similar to the random deposition model ${ }^{22}$. The probability distribution of fluctuations (green data points) is clearly Gaussian, obeying the central limit theorem. Similarly to the previous analysis, all the moments up to the eighth normalized moment are provided and compared with the Gaussian function (solid line) (Supplementary Table 1). This control experiment confirms that the Tracy-Widom distribution of the interface fluctuations results from the coupling between particle positions via laser-induced fluid flows.

The appearance of the Tracy-Widom distribution suggests that the result is robust against experimental imperfections, given that each experiment is noticeably different because the conditions cannot be made entirely identical. Significantly, the combined statistics of our experiments converge to this distribution with an excellent agreement up to the eighth normalized moment. This robustness is likely to have profound physical reasons related to the highly nonlinear and strongly stochastic conditions.

\section{Discussion and conclusions}

Viewed from a broader perspective, the method we report on is related to convective aggregation, which was first described in the seminal paper of von Smoluchowski in $1917^{45}$. More recently, studies in this area have focused on the so-called coffee-ring ${ }^{46}$ and Cheerios $^{47}$ effects to gain better control over aggregation dynamics and inducing long-range arrangements of the particles, specifically at the sub-10-nm scale. Our method departs from all these techniques by the deliberate and precise creation of convection via the femtosecond pulses, which affords us a reasonable control over the size, position and geometry of the aggregates of a wide range of materials.

To give examples, we collected colloidal particles at the beam positions; then, we moved the beam from right to left to form a line out of the aggregate. Moving the beam sinusoidally resulted in a wave pattern of the aggregate (Extended Data Fig. 6 and Supplementary Video 5). It is also possible to structure the laser beam via spatial light modulators to impart more sophisticated, potentially arbitrarily complex forms and motion to the beam and aggregates. For instance, we used a spatial light modulator and computer-generated holographic algorithms (see Supplementary Information for details) to divide our laser beam into multiple beams and structure them to write 'Hi!' and 'bye!' as well as to form triangular, rectangular, circular and star-like shapes (Fig. 3a and Supplementary Video 6). The beam patterns are given in the insets of each image of Fig. 3a, which shows that the collected particles form the same words and shapes. These demonstrations are not specific to colloids; similarly, aggregates of quantum dots and 
living organisms can also be given arbitrary dynamic geometrical shapes. A rotating light rod can attract fluorescent quantum dots as suggested by a narrow ' $\mathrm{X}$ ' shape in Fig. 3b and Supplementary Video 7, which indicates that the dots are collecting quite fast and catching up with the light rod at multiple positions. Figure $3 \mathrm{c}$ and Supplementary Video 7 show an aggregate of yeast cells in a rectangular shape, which can be rotated around its axis.

Quantitative differences of the assembled entities can also be exploited to accomplish nominally difficult tasks with our methodology. As a demonstration, we show separation of E. coli (gramnegative) and $M$. luteus (gram-positive) bacterial cells starting from initially homogeneously mixed populations (Fig. $4 \mathrm{a}$ and Supplementary Video 8): the laser and the direction of beam movement are denoted with a red dot and white arrows. The laser beam was moved up and down, collecting many bacterial cells of both species in the scanned area. E. coli quickly adhered to the glass slide using their fimbriae and flagella whereas $M$. luteus kept floating on top since they lack such adherent parts. Then, we changed the scan pattern of the beam (at 0 min $16 \mathrm{~s}$ of Supplementary Video 8). The $M$. luteus followed the beam and moved away, but the E. coli stayed behind because of their restricted mobility. Naturally, the two species have been separated as a result of their physical differences. It has long been known that gram-negative bacteria can tether on glass slide surfaces via their flagella ${ }^{48}$. Laser-trapped $E$. coli cells are instantaneously attached on glass slides, yet little is known of the molecular mechanisms that mediate attachment ${ }^{49}$. Cyclic dimeric guanylate monophosphate (c-di-GMP), a secondary messenger molecule, has been shown to control the flagellar machinery and transition from planktonic to adherent state ${ }^{50}$. Here, laser-mediated fluid flows may have modified either the chemotaxis signalling or c-di-GMP concentrations inside the bacterial cells to alter their tendency to adhere on the glass surface.

Another example can be seen from Fig. $4 \mathrm{~b}$ and Supplementary Video 9, where we use vertices as vessels to stir and cluster $\sim 30$ yeast cells into the vertex flows: we can start these vertex flows by moving the laser beam, and controllably stop them when we stop moving the beam. Our method is extremely versatile in the sense that it allows the formation of various kinds of fluid flow, for example laminar, shear and vortex, by merely adjusting the relevant parameters.

All these proof-of-principle demonstrations can be further optimized to study micro/nanorobotics by decorating arbitrary nanoparticles with chemicals to functionalize their surfaces to become swimmers, cargo transporters or motors. Manipulation of microorganisms within short periods can also find critical applications in biological processes. For instance, it is possible to collect microorganisms in controlled quantities and at desired spatial positions to scrutinize emergent collective behaviour such as quorum sensing and biofilm formation.

Emergent phenomena in low-dimensional materials, colloidal particles, active matter, microorganisms and cells, to name a few, can be explored since our system is unique in that it operates far from equilibrium. This feature offers the possibility of exploring a broader region of the systems phase space to obtain access to a plethora of configurations, for example different structures, functions and behaviours that are not readily accessible to systems operating near equilibrium or temporarily far from equilibrium. Far-from-equilibrium conditions dictate strong, non-uniform fluctuations, which can be quite useful to investigate dynamic adaptive phenomena. In our earlier work, we showcased this possibility through dynamic bistable colloidal crystals ${ }^{14}$.

Our method can also solve a technical problem in the highyield separation of solution-synthesized low-dimensional systems by collecting large numbers of nanoparticles at a particular position within a few seconds. It is also possible to tile up and 'freeze' these quantum-confined particles on surfaces in non-close-packed arrangements. We showed such arrangements with good uniformity over large areas earlier with colloids ${ }^{14}$. This may be very interesting for, for example, plasmonics, dielectric metasurfaces, sensors and actuators, photovoltaics and light-emitting diodes.

\section{Online content}

Any methods, additional references, Nature Research reporting summaries, source data, extended data, supplementary information, acknowledgements, peer review information; details of author contributions and competing interests; and statements of data and code availability are available at https://doi.org/10.1038/s41567020-0879-8.

Received: 14 October 2019; Accepted: 13 March 2020;

Published online: 20 April 2020

\section{References}

1. Heinen, L. \& Walther, A. Celebrating Soft Matter's 10th anniversary: approaches to program the time domain of self-assemblies. Soft Matter 11, 7857-7866 (2015).

2. Showalter, K. \& Epstein, I. R. From chemical systems to systems chemistry: patterns in space and time. Chaos 25, 097613 (2015).

3. Wojciechowski, J. P., Martin, A. D. \& Thordarson, P. Kinetically controlled lifetimes in redox-responsive transient supramolecular hydrogels. J. Am. Chem. Soc. 140, 2869-2874 (2018).

4. Hu, K. \& Sheiko, S. S. Time programmable hydrogels: regulating the onset time of network dissociation by a reaction relay. Chem. Commun. 54, 5899 (2018).

5. Che, H., Cao, S. \& Van Hest, J. C. M. Feedback-induced temporal control of 'breathing' polymersomes to create self-adaptive nanoreactors. J. Am. Chem. Soc. 140, 5356-5359 (2018).

6. Dhiman, S., Jain, A., Kumar, M. \& George, S. J. Adenosine-phosphate-fueled, temporally programmed supramolecular polymers with multiple transient states. J. Am. Chem. Soc. 139, 16568-16575 (2017).

7. Burute, M. \& Kapitein, L. C. Cellular logistics: unraveling the interplay between microtubule organization and intracellular transport. Annu. Rev. Cell Dev. Biol. 35, 22.1-22.26 (2019).

8. Cheeseman, I. M. \& Desai, A. Molecular architecture of the kinetochoremicrotubule interface. Nat. Rev. Mol. Cell Biol. 9, 33-46 (2008).

9. Bansagi, T., Vanag, V. K. \& Epstein, I. R. Tomography of reaction-diffusion microemulsions reveals three-dimensional Turing patterns. Science 331, 1309-1312 (2011).

10. Yan, J., Bloom, M., Bae, S. C., Luijten, E. \& Granick, S. Linking synchronization to self-assembly using magnetic Janus colloids. Nature 491, 578-581 (2012).

11. Palacci, J., Sacanna, S., Steinberg, A. P., Pine, D. J. \& Chaikin, P. M. Living crystals of light-activated colloidal surfers. Science 339, 936-940 (2013).

12. Aubret, A., Youssef, M., Sacanna, S. \& Palacci, J. Targeted assembly and synchronization of self-spinning microgears. Nat. Phys. 14, 1114-1118 (2018).

13. Alapan, Y., Yigit, B., Beker, O., Demirörs, A. F. \& Sitti, M. Shape-encoded dynamic assembly of mobile micromachines. Nat. Mater. 18, 1244-1251 (2019).

14. Ilday, S. et al. Rich complex behaviour of self-assembled nanoparticles far from equilibrium. Nat. Commun. 8, 14942 (2017).

15. Bisket, G. \& England, J. L. Nonequilibrium associative retrieval of multiple stored self-assembly targets. Proc. Natl Acad. Sci. USA 115, 45 (2018).

16. Prost, J., Julicher, F. \& Joanny, J.-F. Active gel physics. Nat. Phys. 11, 111-117 (2015)

17. Tafoyaa, S., Larged, S. J., Liue, S., Bustamante, C. \& Sivak, D. A Using a system's equilibrium behavior to reduce its energy dissipation in nonequilibrium processes. Proc. Natl Acad. Sci. USA 116, 5920-5924 (2019).

18. Jarzynski, C. Diverse phenomena, common themes. Nat. Phys. 11, 105-107 (2015).

19. Woods, D. Intrinsic universality and the computational power of self-assembly. Phil. Trans. R. Soc. A 373, 20140214 (2015).

20. Woods, D. et al. Diverse and robust molecular algorithms using reprogrammable DNA self-assembly. Nature 567, 366-372 (2019).

21. Lin, M. Y. et al. Universality in colloid aggregation. Nature 339, 360-362 (1989).

22. Barabasi, A.-L. \& Stanley, H. E. Fractal Concepts in Surface Growth (Cambridge University Press, 1995).

23. Tracy, C. A. \& Widom, H. Level spacing distributions and the Airy kernel. Commun. Math. Phys. 159, 151-174 (1994).

24. Tracy, C. A. \& Widom, H. On orthogonal and symplectic matrix ensembles. Commun. Math. Phys. 177, 727-754 (1996). 
25. Majumdar, S. N. \& Schehr, G. Top eigenvalue of a random matrix: large deviations and third order phase transition. J. Stat. Mech. 2014, P01012 (2014).

26. Baik, J. \& Rains, E. M. Limiting distributions for a polynuclear growth model with external sources. J. Stat. Phys. 100, 523-541 (2000).

27. Prahofer, M. \& Spohn, H. Universal distributions for growth processes in $1+1$ dimensions and random matrices. Phys. Rev. Lett. 84, 4882-4885 (2000).

28. Amir, G., Corwin, I. \& Quastel, J. Probability distribution of the free energy of the continuum directed random polymer in $1+1$ dimensions. Commun. Pure Appl. Math. 64, 466-537 (2011).

29. Biroli, G., Bouchaud, J.-P. \& Potters, M. On the top eigenvalue of heavy-tailed random matrices. Eur. Phys. Lett. 78, 10001 (2007).

30. Halpin-Healy, T. \& Zhang, Y. C. Kinetic roughening phenomena, stochastic growth, directed polymers and all that. Phys. Rep. 254, 215-414 (1995).

31. Takeuchi, K. A. \& Sano, M. Universal fluctuations of growing interfaces: evidence in turbulent liquid crystals. Phys. Rev. Lett. 104, 230601 (2010).

32. Miettinen, L., Myllys, M., Merikoski, J. \& Timonen, J. Experimental determination of KPZ height-fluctuation distributions. Eur. Phys. J. B 46, 55-60 (2005).

33. Fridman, M., Pugatch, R., Nixon, M., Friesem, A. A. \& Davidson, N. Measuring maximal eigenvalue distribution of Wishart random matrices with coupled lasers. Phys. Rev. E 85, 020101(R) (2012).

34. Vogel, A., Linz, N., Freidank, S. \& Paltauf, G. Femtosecond-laser-induced nanocavitation in water: implications for optical breakdown threshold and cell surgery. Phys. Rev. Lett. 100, 038102 (2008).

35. Haken, H. Synergetics, an Introduction: Nonequilibrium Phase Transitions and Self-Organization in Physics, Chemistry, and Biology 3rd edn (Springer, 1983).

36. Darcy, H. Les Fontaines Publiques de la Ville de Dijon (Dalmont, 1856).

37. Vallejo, D. M., Juarez-Carreño, S., Bolivar, J., Morante, J. \& Dominguez, M. A brain circuit that synchronizes growth and maturation revealed through Dilp8 binding to Lgr3. Science 350, aac6767 (2015).

38. Lindsay, R. J., Pawlowska, B. J. \& Gudelj, I. Privatization of public goods can cause population decline. Nat. Ecol. Evol. 3, 1206-1216 (2019).
39. Chen, Y.-G. Logistic models of fractal dimension growth of urban morphology. Fractals 26, 1850033 (2018).

40. Kardar, M., Parisi, G. \& Zhang, Y.-C. Dynamic scaling of growing interfaces. Phys. Rev. Lett. 56, 889-892 (1986).

41. Aldous, D. \& Diaconis, P. Hammersley's interacting particle process and longest increasing subsequences. Probab. Theory Relat. Fields 103, 199-213 (1995).

42. Chiani, M. Distribution of the largest eigenvalue for real Wishart and Gaussian random matrices and a simple approximation for the Tracy-Widom distribution. J. Multivar. Anal. 129, 69-81 (2014).

43. Baik, J., Deift, P. \& Johansson, K. On the distribution of the length of the longest increasing subsequence of random permutations. J. Am. Math. Soc. 12, 1119-1178 (1999).

44. Aldous, D. \& Diaconis, P. Longest increasing subsequences: from patience sorting to the Baik-Deift-Johansson theorem. Bull. Am. Math. Soc. 36, 413-432 (1999).

45. von Smoluchowski, M. Versucheiner mathematischen theorie der koagulations kinetic kolloider lousungen. Z. Phys. Chem. 92, 129-168 (1917).

46. Deegan, R. D. et al. Capillary flow as the cause of ring stains from dried liquid drops. Nature 389, 827-829 (1997).

47. Vella, D. \& Mahadevan, L. The "Cheerios effect". Am. J. Phys. 72, 817-825 (2005).

48. Meadows, P. S. The attachment of bacteria to solid surfaces. Arch. Mikrobiol. 75, 374-381 (1971).

49. Jones, J. F. \& Velegol, D. Laser trap studies of end-on E. coli adhesion to glass. Colloids Surf. B 50, 66-71 (2006).

50. Fang, X. \& Gomelsky, M. A post-translational, c-di-GMP-dependent mechanism regulating flagellar motility. Mol. Microbiol. 76 , 1295-1305 (2010)

Publisher's note Springer Nature remains neutral with regard to jurisdictional claims in published maps and institutional affiliations.

(c) The Author(s), under exclusive licence to Springer Nature Limited 2020 


\section{Methods}

Preparation of quantum dots and colloids. Luminescent aqueous $\mathrm{CdTe}$ quantum dots $(\sim 2.5-3.5 \mathrm{~nm}$ in size) were stabilized in thioglycolic acid and synthesized in deionized water following the recipe reported in ref. ${ }^{51}$, which was modified from the protocol reported in ref. ${ }^{52}$. After mixing $4.59 \mathrm{~g}$ of $\mathrm{Cd}\left(\mathrm{ClO}_{4}\right)_{2}$ (prepared in 0.21 of deionized water) and $1.31 \mathrm{gl}^{-1}$ thioglycolic acid (prepared in 0.31 of deionized water) solutions, $1 \mathrm{M} \mathrm{NaOH}$ was added under vigorous stirring to set the $\mathrm{pH}$ of the mixture to 12. The mixture was then flushed with Ar and kept under Ar atmosphere. Subsequently, $0.8 \mathrm{~g}$ of $\mathrm{Al}_{2} \mathrm{Te}_{3}$ was placed in a second flask in a glovebox. Separately, a solution of $10 \mathrm{ml}$ of $0.5 \mathrm{M} \mathrm{H}_{2} \mathrm{SO}_{4}$ and $15 \mathrm{ml}$ of deionized water was prepared. The second flask was then connected to the main flask, and $10 \mathrm{ml}$ of the prepared $\mathrm{H}_{2} \mathrm{SO}_{4}$ solution was slowly added to the second flask containing $\mathrm{Al}_{2} \mathrm{Te}_{3}$. Ar was used as the carrier gas during the reaction. $\mathrm{The}_{2} \mathrm{Al}_{2} \mathrm{Te}_{3}$ line was disconnected after $30 \mathrm{~min}$, and a cooler was connected to the system. The heater was set to reach $100^{\circ} \mathrm{C}$. Upon boiling of the solution, colloidal CdTe quantum dots started to form. Before using synthesized quantum dots in the experiments, they were purified by evaporating about $90 \%$ of the solvent in a rotary evaporator. The remaining highly concentrated dispersion was sonicated and centrifuged. Finally, the precipitate was dissolved, recentrifuged and stored in the dark in the refrigerator. A transmission electron microscopy image and photoluminescence spectrum are shown in Extended Data Fig. 7.

Pure polystyrene colloidal spheres $497 \pm 7 \mathrm{~nm}$ in diameter were purchased from Microparticles and $\sim 150$ - $\mu \mathrm{m}$-thick optically transparent glass slides were purchased from ISOLAB Laborgeräte.

\section{Preparation of microorganisms. Microorganisms used in this study were}

- E. coli K12 RP437 with the genotype F-, thr-1, araC14, leuB6(Am), fhuA31, lacY1, tsx-78, $\lambda$-, eda-50, hisG4(Oc), rfbC1, rpsL136(strR), xylA5, mtl-1, metF159(Am), thiE1 $1^{53}$

- M. luteus (Schroeter) Cohn (ATCC 4698) ${ }^{54}$ and S. cerevisiae InvSc1 (ThermoFisher Scientific, Invitrogen C81000) (MATa his3D1 leu2 trp1-289 ura3-52 MAT his3D1 leu2 trp1-289 ura3-52).

Cultures for E. coli and M. luteus were grown to mid-logarithmic phase $\left(0.6 \mathrm{OD}_{600}\right.$, optical density at $\left.600 \mathrm{~nm}\right)$ in Luria broth medium containing $10 \mathrm{gl}^{-1}$ pancreatic digest of casein, $5 \mathrm{gl}^{-1} \mathrm{NaCl}$ and $5 \mathrm{gl}^{-1}$ yeast extract at $33^{\circ} \mathrm{C}\left(\right.$ ref. ${ }^{55}$ ). $S$. cerevisiae cultures were grown to $0.8 \mathrm{OD}_{600}$ in yeast extract peptone dextrose broth containing $20 \mathrm{gl}^{-1}$ peptone, $20 \mathrm{gl}^{-1}$ glucose and $10 \mathrm{gl}^{-1}$ yeast extract at $30^{\circ} \mathrm{C}$ (refs. ${ }^{56,57}$ ). Just before the experiments, microbial cells were harvested by centrifugation at 4,000 r.p.m. for $5 \mathrm{~min}$ at $25^{\circ} \mathrm{C}$. Motility buffer containing $10 \mathrm{mM}$ potassium phosphate buffer at $\mathrm{pH} 7.0,0.1 \mathrm{mM}$ EDTA and $10 \mathrm{mM}$ glucose was used to wash the cells. Following repeated centrifugation steps, cells were resuspended in motility buffer ${ }^{58}$.

Preparation of human cells. The MCF10A normal breast cell line was obtained from ATCC (Manassas, VA, USA) and cultured in Dulbecco's modified Eagle's medium (Lonza, NJ, USA) supplemented with 10\% fetal bovine serum (Lonza), $1 \%$ non-essential amino acids (Gibco, Carlsbad, CA), $50 \mathrm{U} \mathrm{ml}^{-1}$ penicillin/streptomycin (Gibco), $20 \mathrm{ng} \mathrm{ml}^{-1}$ epidermal growth factor, $500 \mathrm{ng} \mathrm{ml}^{-1}$ hydrocortisone and $0.1 \%$ insulin (Sigma-Aldrich, MO, USA). The cells were maintained in 100-mm tissue culture dishes at $37^{\circ} \mathrm{C}$ in an atmosphere of $5 \% \mathrm{CO}_{2}$. They were passaged with trypsin (Lonza) upon becoming confluent. The presence of mycoplasma in all cell lines was tested regularly with a MycoAlert mycoplasma detection kit (Lonza). Before experiments, cells were synchronized through serum starvation ${ }^{59}$. First, cells were counted and seeded into six-well plates $\left(2 \times 10^{5}\right.$ cells per well $)$ in their growth medium with all necessary supplements. To synchronize cells at the G1 stage, we incubated cells in media without fetal bovine serum for $24 \mathrm{~h}$ and collected the cells for further analysis. G1 arrest was determined by the decrease of cyclin D1 levels ${ }^{60,61}$ by western blot analysis. The solution of MCF10A cells $(\sim 15 \mu \mathrm{m}$ in diameter) was adjusted to $\sim 250,000$ cells per $100 \mu$ in their growth media.

All experiments on living organisms were performed in the presence of trypan blue dye (Sigma-Aldrich) to assess cell viability.

Growth-curve analysis of the aggregates. To analyse the temporal evolution of the aggregates, we traced individual particles/organisms forming the aggregate in a finite area following the steps detailed in our previous study ${ }^{14}$. However, we modified our particle tracking algorithm for each entity because of the variations in their sizes and geometries as detailed below.

- For polystyrene colloids we used the data from our previous study ${ }^{14}$

- For MCF10A and S. cerevisiae cells, we counted the numbers of cells manually during aggregate formation because cell sizes were large and the number of cells forming aggregates was relatively small ( $\sim 15-20$ cells per experiment), enough to be detected individually by the human eye with the aid of video editing software (Adobe's After Effect CC 2019) that allows access to a highly accurate timeline for the temporal accuracy of cell counting.

- For E. coli and M. luteus cells, we had to modify our algorithm because E. coli cells are anisotropic and $M$. luteus cells have soft bodies that are difficult to detect. Therefore, we set a rectangular window that covers the largest detectable area of the aggregate (Extended Data Fig. 8). Next, we traced the outline of aggregation within this window semimanually using Adobe After Effect software at manually selected frames. The traced outline was then used to form a binary dynamic mask tracking the area growth of the aggregate in the intermediate frames between two manually selected frames. This semimanual outlining was necessary since the software was unable to track individual cells correctly over the entire video without human aid. Fully manual tracking was also not possible due to the large number of video frames. Finally, we processed the dynamic mask through a MATLAB routine, which calculated the areal growth of the aggregation by counting the number of active pixels in the binary mask within the rectangular window (Supplementary Video 10).

- $\quad$ For the quantum dots, we could not trace the individual quantum dots because their sub-diffraction-limited sizes preclude direct optical observation. Instead, we used fluorescent quantum dots and tracked the intensity of photoluminescence generated by accumulating particles (Extended Data Fig. 9). We uniformly applied incoherent excitation light across the sample to induce photoluminescence, which was detected optically via an electron-multiplying CCD (charge-coupled device) camera. Narrow-band-pass chromatic filters (FB580-10 and FES 750) were applied to pass the photoluminescence signal to camera pixels, which is proportional to the density of particles at the position corresponding to each pixel. Background signal due to dark current and stray light were subtracted from the photoluminescence signal, which was then integrated over the area to represent the aggregation size (Extended Data Fig. 9). Finally, we applied gentle smoothing to the integrated curve to minimize the digitization noise due to finite voltage detection bits from the electronics of the camera (Extended Data Fig. 9).

The individual growth curves for all particles/organisms can be seen in Extended Data Fig. 3. In the figure the time axis of each plot is different because in each case the applied laser power, and hence the velocity of the flow and speed of collection, are different. We cannot use the same laser power for, say, polystyrene spheres and human cells because the properties of their liquid media, for example viscosity, surface tension and capillary action, are quite different. Therefore, we have to adjust the power so that the control over the bubbles and flows is not hampered.

To compare the individual aggregate growth curves for each entity, we applied vertical rescaling so that the filling ratios of individual aggregates ranges between 0 and 1 . Then, each of these curves was fitted with a sigmoidal curve (the logistic function), described by

$$
f(t)=\frac{1}{1+\mathrm{e}^{-(a t+b)}}
$$

where $a$ and $b$ correspond to the time constant and shift in time, respectively. The speeds of aggregation for different entities are characterized by $a$ and the origin of time is set by $b$. These values were determined by fitting each curve using MATLAB's curve fitting toolbox. Next, the time axes were shifted and rescaled to have $a=1$ and $b=0$.

Interface detection algorithm. We recorded 14 videos of circularly growing aggregates at 300 frames per second. Tracking the individual particles by manual processing was not possible due to the high particle densities and sheer number of data. Therefore, we based our detection of growing interfaces (perimeter) on the relative stillness of the particles inside an aggregate in comparison with those outside (Supplementary Video 3), using the following procedure.

- First, light intensity in each video was normalized. The standard deviation (s.d.) values of the light intensity for every pixel in every frame of all 14 videos were calculated and compared using a certain number of preceding frames (hereinafter referred to as the 'span', which we set to be equal to 100 frames), similarly to how a running average is calculated. By doing so, we formed three-dimensional (3D) arrays to describe the pixel-wise evolution of the s.d. of light intensities at each pixel, one 3D array for each of the 14 videos. To suppress the camera-associated pixel noise, $2 \mathrm{D}$ matrices corresponding to each frame (that is, $2 \mathrm{D}$ slices of a $3 \mathrm{D}$ array of s.d. values) were passed through a gentle 2D Gaussian filter (with a narrow smoothing kernel). After this step, s.d. values of light intensities can be used as reliable indicators for the displacement of particles.

- Next, dynamic masks were generated to define the boundaries of growing aggregates by applying a threshold number to describe the amount of displacement for each particle at each pixel. This threshold number was chosen empirically to ensure reliable detection of the boundaries. The thresholding operation was applied uniformly to every pixel. Values lower than this threshold imply that the particle at that pixel is largely immobilized, ergo part of the aggregate, so the pixel was assigned a value of 1 for the mask. Values higher than this threshold imply that the particle at that pixel is mobile, outside the aggregate, so the pixel was assigned a value of 0 for the mask.

- On occasion, we detected empty pixels devoid of particles (that is, pixels in between two particles) that did not display any motion regardless of them being inside or outside the aggregate. The thresholding operation then creates an artificially granular boundary. To solve this problem, we adjusted our algorithm to detect protuberances along the granular boundary using the morpho- 
logical operators technique ${ }^{62}$, namely image close and image open operators. These operators were set to fill the space along the edges of mask using discs of the same size as the particles (close operator) if they fitted this space. Then, a morphological operator (open operator) was applied to eliminate remaining protuberances smaller than the particle size. Dynamic masks obtained this way were binary image sequences without any granular noise.

After the described procedure, we obtained the refined dynamic masks that accurately describe the boundaries of growing aggregates for Tracy-Widom analysis.

Tracy-Widom analysis. To determine the height values of circularly growing aggregates, distances from each pixel along the detected aggregate boundaries to their centroids were calculated. The edges were detected using a Sobel edgedetection algorithm ${ }^{63}$ and centroids were calculated as the centre of mass of the mask using the built-in MATLAB functions ${ }^{64}$. The height values were then calculated along the aggregate boundary for each pixel of all frames for 14 videos using the following equation ${ }^{22,30,31}$,

$$
h\left(u_{n}, t\right)=\sqrt{\left(x_{n}(t)-x_{\mathrm{c}}(t)\right)^{2}+\left(y_{n}(t)-y_{\mathrm{c}}(t)\right)^{2}}
$$

where $u_{n}$ is is the vector connecting a given point, $n$, along the boundary to the centroid (Fig. 3a), $n$ is a pixel index defining the position along the boundary, $\left(x_{n}, y_{n}\right)$ are the coordinates of a pixel on the boundary, $\left(x_{c}, y_{c}\right)$ are the coordinates of the centroids and $t$ is time. Average height, $h_{\text {average }}(t)$, was calculated over the entire aggregate boundary to obtain a single height value per frame,

$$
h_{\text {average }}(t)=\sum_{n=1}^{N(t)} \frac{h\left(u_{n}, t\right)}{N(t)}
$$

where $N(t)$ is the total number of pixels at the boundary at time $t$.

Aggregates were growing with time, so the temporal evolution of height values had the form of a slow growth accompanied by rapid fluctuations, $\chi(t)$. Because it is the latter that is of interest here, we subtracted the growth component by highpass filtering the data. Such filtering is mathematically equivalent to subtracting from the data its running average with a certain temporal span. We note that the span is equivalent to the inverse of cutoff frequency in the high-pass filtering interpretation save for a constant that is of the order of unity $y^{65}$. The span value or equivalently the inverse cutoff frequency has to be chosen small enough that the slow growth is extracted, and not too high, to ensure that fluctuations are retained. To set this value, we plotted the variation of the moments of the temporal fluctuations normalized to those of the Tracy-Widom GUE as a function of the temporal span (Extended Data Fig. 10). Over a fairly broad range, from $\sim 650 \mathrm{~ms}$ to $\sim 850 \mathrm{~ms}$, the normalized moments of the experimental data agree equally well with that of the Tracy-Widom GUE up to eighth normalized moments. Within this range, we chose the span value to be $750 \mathrm{~ms}$. Finally, the fluctuations were normalized and shifted according to the Tracy-Widom GUE probability distribution for each video. Measured fluctuations from 14 videos of similar but different experiments were combined. For each time instant, that is, each video frame, the average heights were calculated over several hundred individual height values along the respective boundary. In total, we have used boundaries detected from approximately 21,000 frames. For statistical analysis, lowest-order moments, namely sample mean and sample s.d., were calculated, as follows, respectively:

$$
\begin{gathered}
\mu=\frac{1}{M} \sum_{m=1}^{M} x_{m} \\
\sigma=\left(\frac{1}{M} \sum_{m=1}^{M}\left|x_{m}-\mu\right|^{2}\right)^{1 / 2}
\end{gathered}
$$

The higher moments were calculated as the normalized central moments (normalized by s.d.) and are defined as follows:

$$
C_{n}=\frac{1}{M} \frac{\sum_{m=1}^{M}\left|x_{m}-\mu\right|^{n}}{\sigma^{n}}
$$

The exact values of the higher moments ( $>4$ th) of the Tracy-Widom distribution are not known. Hence we used a simple approximation for the TracyWidom GUE distribution ${ }^{44}$. For the control experiment, we counted particles passing through a virtual boundary and formed records of how many particles had passed through each point along this boundary. Our counting algorithm was based on Hough-transform detection, and is explained in detail in ref. ${ }^{14}$. Upon seeing that the fluctuations followed a Gaussian distribution, they were normalized and shifted according to the Gaussian scaling.

Numerical generation of Tracy-Widom statistics. For numerical generation of finite-size samples that are formally guaranteed to converge to the Tracy-Widom GUE distribution in the limit of large samples, we consider two models that describe physically very different scenarios but are mathematically equivalent.
The first model is the LIS model for uniform random permutations. Recall that any arrangement of the elements of $[\mathbf{n}]:=\{1, \ldots, n\}$ is called a permutation. We use the notation $\sigma=\sigma_{1} \sigma_{2} \cdots \sigma_{n}$ to denote a permutation of [n]. Let $L_{n}(\sigma)$ be the length of an LIS in $\sigma$, that is,

$$
\begin{array}{r}
L_{n}(\sigma)=\max \left\{k \in[\mathbf{n}]: \text { there exist } 1 \leq i_{1}<i_{2}<\ldots\right. \\
\left.<i_{k} \leq n \text { such that } \sigma_{i_{1}}<\sigma_{i_{2}}<\ldots<\sigma_{i_{k}}\right\}
\end{array}
$$

In general, such a subsequence is not unique. The Erdős-Szekeres theorem ${ }^{66}$ states that every permutation of length $n \geq(r-1)(s-1)+1$ contains either an increasing subsequence of length $r$, or a decreasing subsequence of length $s$. After this result, many researchers worked on the problem of determining the asymptotic behaviour of $L_{n}$ on $S_{n}$, the set of all permutations of length $n$, under the uniform probability distribution. It was rigorously shown that the expected value of $L_{n}$, $E\left(L_{n}\right)$, asymptotically grows as $2 \sqrt{n}\left(\right.$ refs. $\left.{ }^{67-69}\right)$. A real breakthrough was achieved by Baik, Deift and Johansson ${ }^{43}$, who completely determined the asymptotic distribution of $L_{n}$.

Theorem. Consider $S_{n}$ with the uniform probability measure. Then,

$$
\lim _{n \rightarrow \infty} \operatorname{Pr}\left(\frac{L_{n}-2 \sqrt{n}}{n^{1 / 6}} \leq t\right)=F_{\mathrm{TW}}(t) \text { for all } t \in \mathbb{R}
$$

where $F_{\mathrm{TW}}$ is the Tracy-Widom GUE distribution function ${ }^{23,24}$. Moreover,

$$
E\left(\frac{L_{n}-2 \sqrt{n}}{n^{1 / 6}}\right)^{k} \rightarrow \int_{-\infty}^{\infty} x^{k} \mathrm{~d} F_{\mathrm{TW}}(x) \text { as } n \rightarrow \infty
$$

for any positive integer $k$. The integral notation indicates data $x$ is a real number. It follows from Hammersley's work ${ }^{70}$ that there is an interacting-particle process on the unit interval in which the macroscopic quantity defined as the number of particles in the system has the same statistical distribution as the random variable $L_{n}$. The particle process approach also gives a very efficient and elegant algorithm for simulating $L_{n}$.

In the Hammersley process, initially there are zero particles in the system. At each step, a particle appears at a uniform random point $u$ in the interval $[0,1]$. Simultaneously, the nearest particle (if any) to the right of $u$ disappears. If $p_{n}$ denotes the number of particles in the system after $n$ steps, then $p_{n}$ and $L_{n}$ have the same probability distribution, hence the large-time behaviour of the particle system follows the Tracy-Widom GUE distribution.

The correspondence between the particle process and the LISs for permutations readily follows from the patience sorting algorithm with the greedy strategy. We consider a permutation as a shuffled deck of cards numbered from 1 to $n$. The cards are dealt one by one into a sequence of piles, according to the following rules. (1) The first card dealt forms the first pile consisting of the single card. (2) Each subsequent card is placed on the leftmost existing pile whose top card has a value greater the new card's value, or to the right of all of the existing piles, thus forming a new pile.

It follows from the following lemma that the number of piles produced by this algorithm applied to the $n$ cards is equal to the length of the LIS in the corresponding permutation and also to the number of particles in the system in Hammersley's process. Numerical values were generated by a straightforward implementation of this algorithm in MATLAB.

Lemma. Let $\sigma$ be a permutation of length $n$. If patience sorting with the greedy strategy applies to $\sigma$, it ends with exactly $L_{n}(\sigma)$ piles. The greedy strategy is optimal and cannot be improved by any look-ahead strategy.

Reporting Summary. Further information on research design is available in the Nature Research Reporting Summary linked to this article.

\section{Data availability}

The data represented in Fig. 2, Supplementary Fig. 2 and Extended Data Figs. 3, 4, $5,7,8$ and 10 are available as Source Data. Additional data may be requested from the corresponding author.

\section{Code availability}

MATLAB codes used to compute simulations of the Hammersley process and the length of the longest increasing subsequence for a given permutation, TracyWidom GUE simulations, image processing, and holographic algoritms are available from the authors on reasonable request.

\section{References}

51. Ozel, T. et al. Anisotropic emission from multilayered plasmon resonator nanocomposites of isotropic semiconductor quantum dots. ACS Nano $\mathbf{5}$, 1328-1334 (2011)

52. Rogach, A. L. et al. Aqueous synthesis of thiol-capped CdTe nanocrystals: state-of-the-art. J. Phys. Chem. C 111, 14628-14637 (2007). 
53. Parkinson, J. S. \& Houts, S. E. Isolation and behavior of Escherichia coli deletion mutants lacking chemotaxis functions. J. Bacteriol. 151 106-113 (1982).

54. Fleming, A. On a remarkable bacteriolytic element found in tissues and secretions. Proc. R. Soc. Lond. B 93, 306-317 (1922).

55. Atlas, R. M. in Handbook of Microbiological Media 4th edn, 934 (Taylor and Francis, 2010)

56. Atlas, R. M. in Handbook of Microbiological Media 4th edn, 1949 (Taylor and Francis, 2010).

57. Olivares-Marin, I. K., González-Hernández, J. C., Regalado-Gonzalez, C. \& Madrigal-Perez, L. A. Saccharomyces cerevisiae exponential growth kinetics in batch culture to analyze respiratory and fermentative metabolism. J. Vis. Exp. (139), e58192 (2018)

58. Swiecicki, J.-M., Sliusarenko, O. \& Weibel, D. B. From swimming to swarming: Escherichia coli cell motility in two-dimensions. Integr. Biol. 5, 1490-1494 (2013).

59. Hasan, N. M., Adams, G. E. \& Joiner, M. C. Effect of serum starvation on expression and phosphorylation of PKC-alpha and p53 in V79 cells: implications for cell death. Int. J. Cancer 80, 400-405 (1999).

60. Masamha, C. P. \& Benbrook, D. M. Cyclin D1 degradation is sufficient to induce G1 cell cycle arrest despite constitutive expression of cyclin E2 in ovarian cancer cells. Cancer Res. 69, 6565-6572 (2009).

61. Rezaei, P. F., Fouladdel, S., Ghaffari, S. M., Amin, G. \& Azizi, E. Induction of G1 cell cycle arrest and cyclin D1 down-regulation in response to pericarp extract of Baneh in human breast cancer T47D cells. Daru 20, 101 (2012).

62. Gonzalez, R. C., Woods, R. E. \& Eddins, S. L. Digital Image Processing using MATLAB (McGraw-Hill, 2013)

63. Najarian, K. \& Splinter, R. Biomedical Signal and Image Processing (CRC Press, 2016).

64. Marques, O. Practical Image and Video Processing Using MATLAB (Wiley, 2011).

65. King, A. \& Aljabar, P. MATLAB Programming for Biomedical Engineers and Scientists (Academic, 2017).

66. Erdős, P. \& Szekeres, G. A combinatorial theorem in geometry. Compositio Math. 2, 463-470 (1935).

67. Logan, B. F. \& Shepp, L. A. A variational problem for random Young tableaux. Adv. Math. 26, 206-222 (1977).
68. Seppalainen, T. A microscopic model for the Burgers equation and longest increasing subsequences. Electron. J. Probab. 1, 1-51 (1994).

69. Vershik, A. M. \& Kerov, S. V. Asymptotics of the Plancherel measure of the symmetric group and the limiting form of Young tableaux. Sov. Math. Dokl. 18, 527-531 (1977).

70. Hammersley, J. M. A few seedlings of research. In Proc. 6th Berkeley Symposium on Mathematical Statistics and Probability Vol. 1, 345-394 (University of California Press, 1972).

\section{Acknowledgements}

This work received funding from the European Research Council (ERC) under the European Union's Horizon 2020 research and innovation programme (grant agreement 853387), TÜBİTAK under projects $115 \mathrm{~F} 110$ and 117F352, and a L'Oréal-UNESCO FWIS award. F.Ö.I., G.M. and H.V.D. gratefully acknowledge funding from the ERC Consolidator Grant ERC-617521 NLL, TÜBİTAK under project 117E823, and NRF-NRF 1-2016-08 and TÜBA, respectively.

\section{Author contributions}

S.I. designed the research and wrote the paper. S.I., S.G., R.G., G.M., Ö.Y. and O.B. performed the experiments. G.M. carried out image processing. G.M., O.B. and F.Ö.I. performed statistical analysis. Ü.S.N. carried out the numerical simulations of fluid dynamics. G.Y. provided the MATLAB code for the mathematical models. E.D.E. prepared the microorganisms. Ö.A. and Ö.Ş. prepared the human cells. K.G., D.D. and H.V.D. prepared the quantum dots.

\section{Competing interests}

The authors declare no competing interests.

\section{Additional information}

Extended data is available for this paper at https://doi.org/10.1038/s41567-020-0879-8. Supplementary information is available for this paper at https://doi.org/10.1038/ s41567-020-0879-8.

Correspondence and requests for materials should be addressed to S.I.

Peer review information Nature Physics thanks Gili Bisker and the other, anonymous, reviewer(s) for their contribution to the peer review of this work.

Reprints and permissions information is available at www.nature.com/reprints 
a

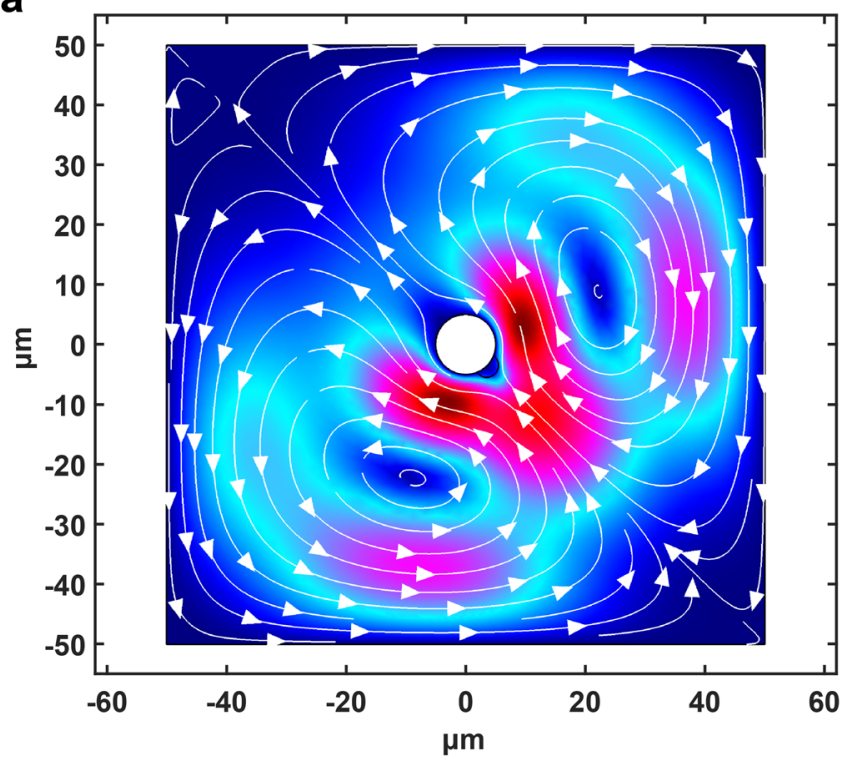

b

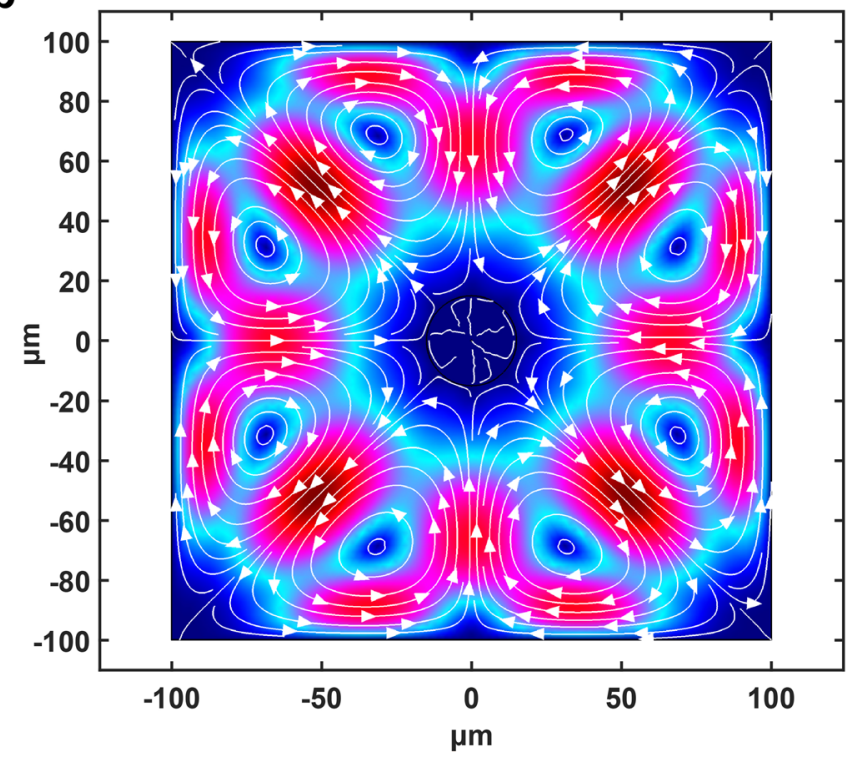

Extended Data Fig. 1 | Numerical simulation of the fluid flows. Numerical simulation results show velocity field distribution of the fluid flows and corresponding streamlines (a) in the presence and (b) in the absence of a cavitation bubble. Red (dark blue) denotes the highest (lowest) flow speeds. The cavitation bubble is depicted by the white circle located at the centre of the computational area. The effect of the laser pulses is modelled as a boundary heat source at the lower right quarter of the bubble. A porous medium was introduced to model the aggregate positioned adjacent to the lowerright quarter of the bubble. In the absence of a bubble, we described the aggregate, also as a porous medium, located at the centre of the computational area (black circle). The beam profile of the laser is Gaussian, so we introduced a heat source with a Gaussian temperature profile to represent the effect of the laser. This source was located at the centre of the aggregate. The diameters of the porous medium and the laser beam were chosen to be $15 \mu \mathrm{m}$ and $8 \mu \mathrm{m}$. The streamlines can be seen to penetrate the aggregate because it is modelled as a porous medium. 

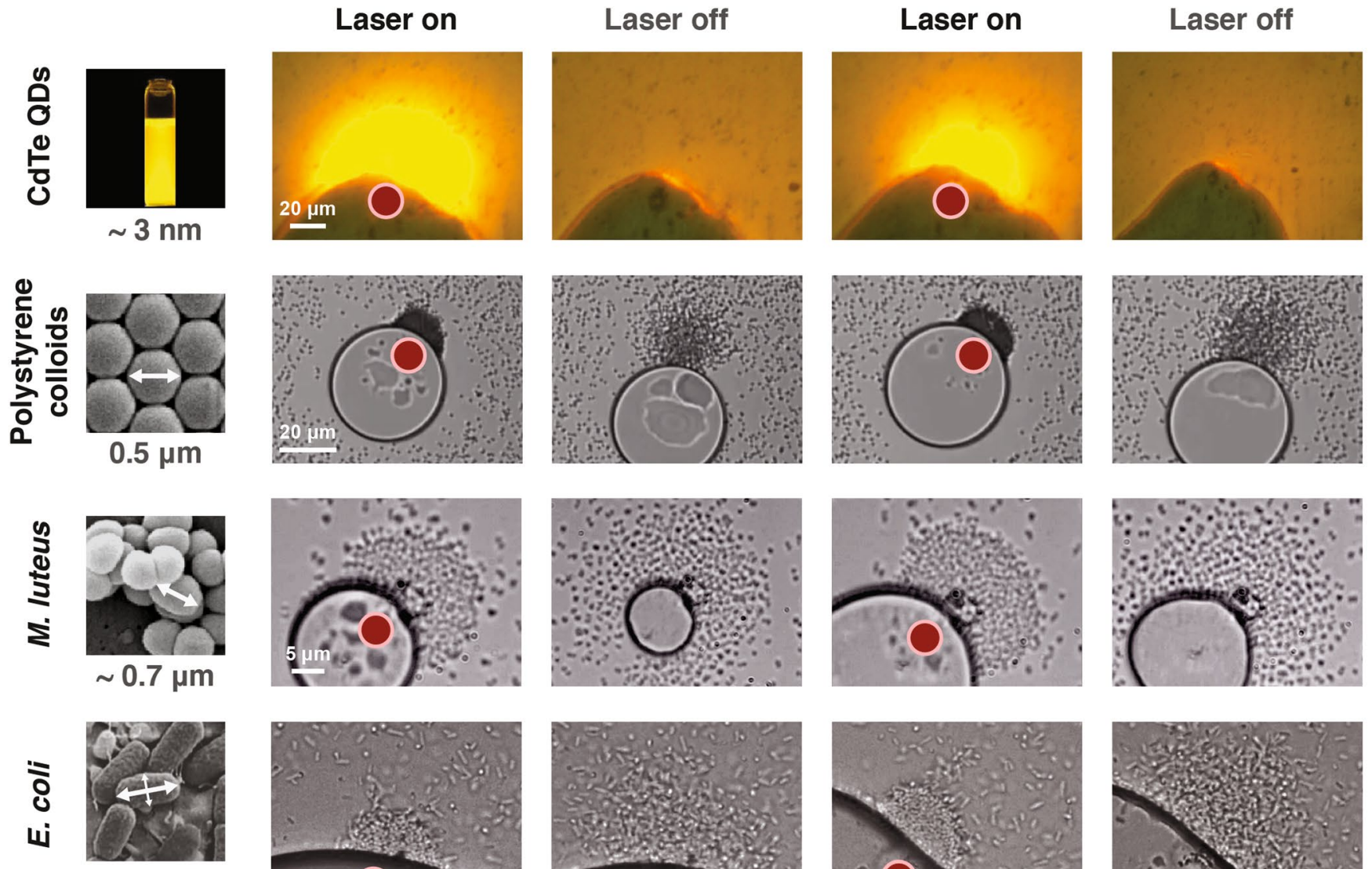

$\sim 1 \mu \mathrm{m} \times 2 \mu \mathrm{m}$
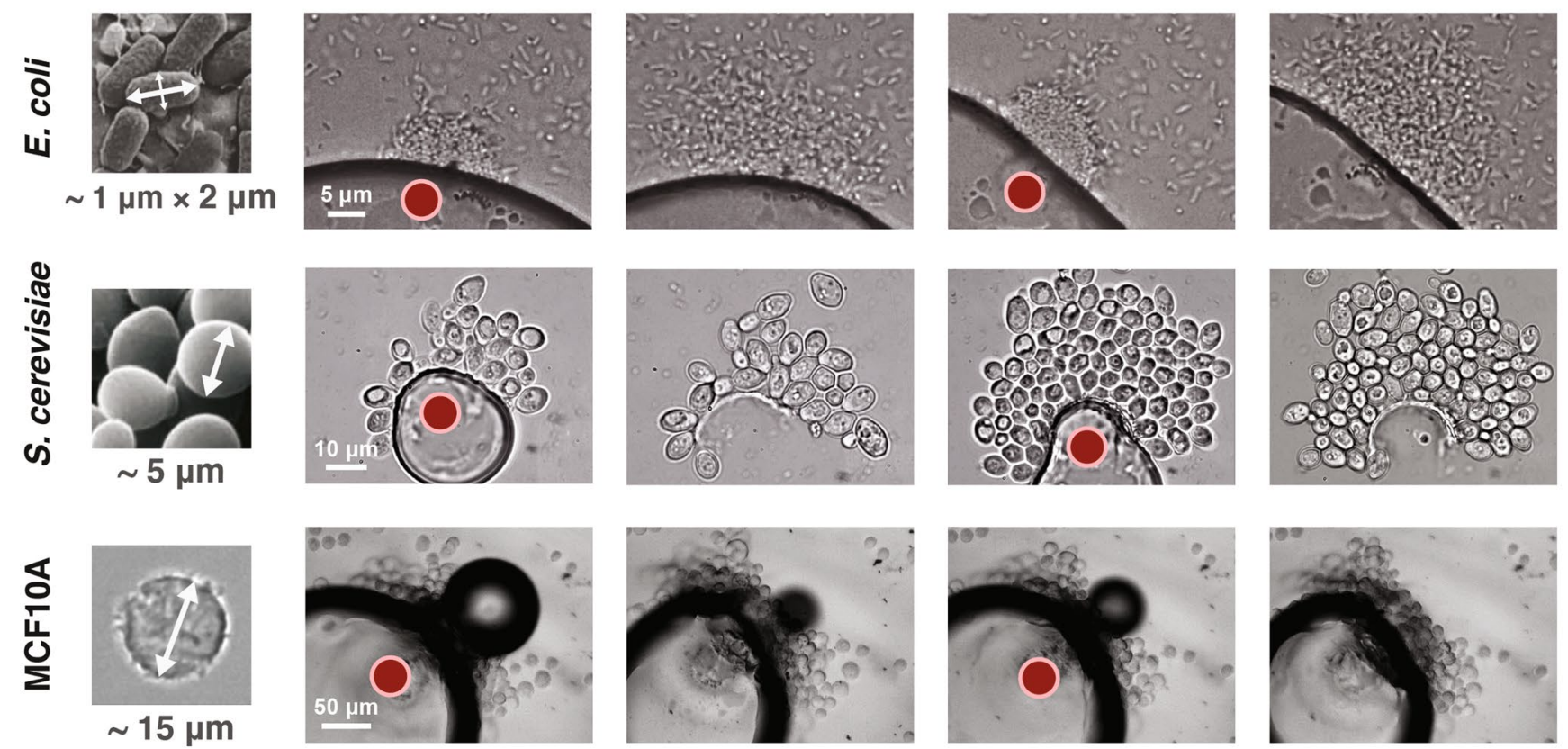

Position of laser beam

Extended Data Fig. 2 | Microscopy images of aggregate collection and dissolution. Microscopy images showing collection (laser on) and dissolution (laser off) of the aggregates of the particles and organisms. Red dot denotes position of the laser beam. Beam sizes are not drawn to scale; it is fixed to be $\sim 8 \mu \mathrm{m}$ in all experiments. A $\times 40$ objective was used for imaging the $\mathrm{CdTe}$ quantum dots, polystyrene spheres, and S. cerevisiae yeast cells, whereas a $\times 100$ objective was used for M. Luteus and E. Coli bacterial cells and $\times 10$ for MCF10A human cells. 

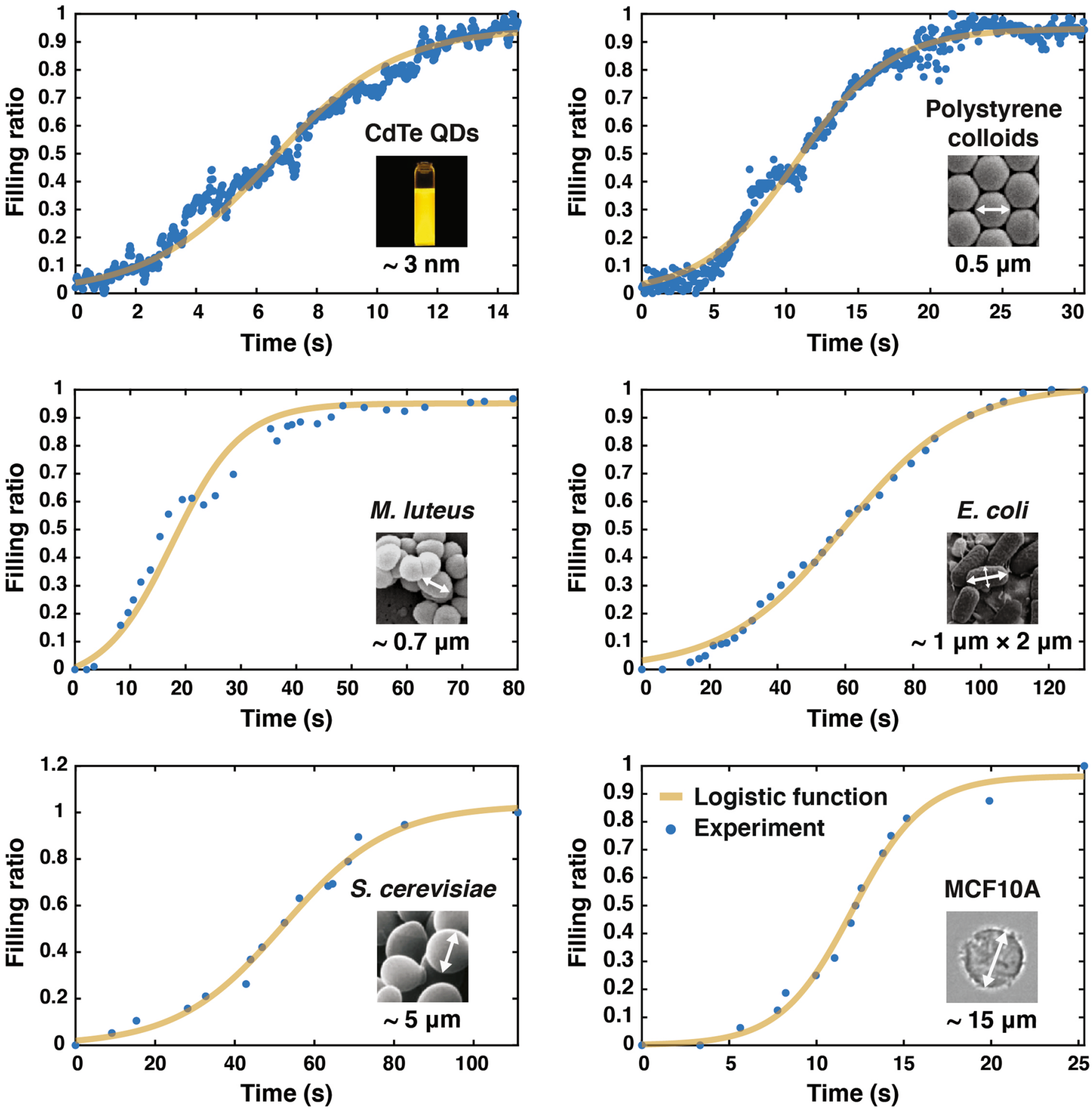

Extended Data Fig. 3 | Growth curves at original time scales. Graphs showing individual growth curves of particles and living organisms at their original time scales. 


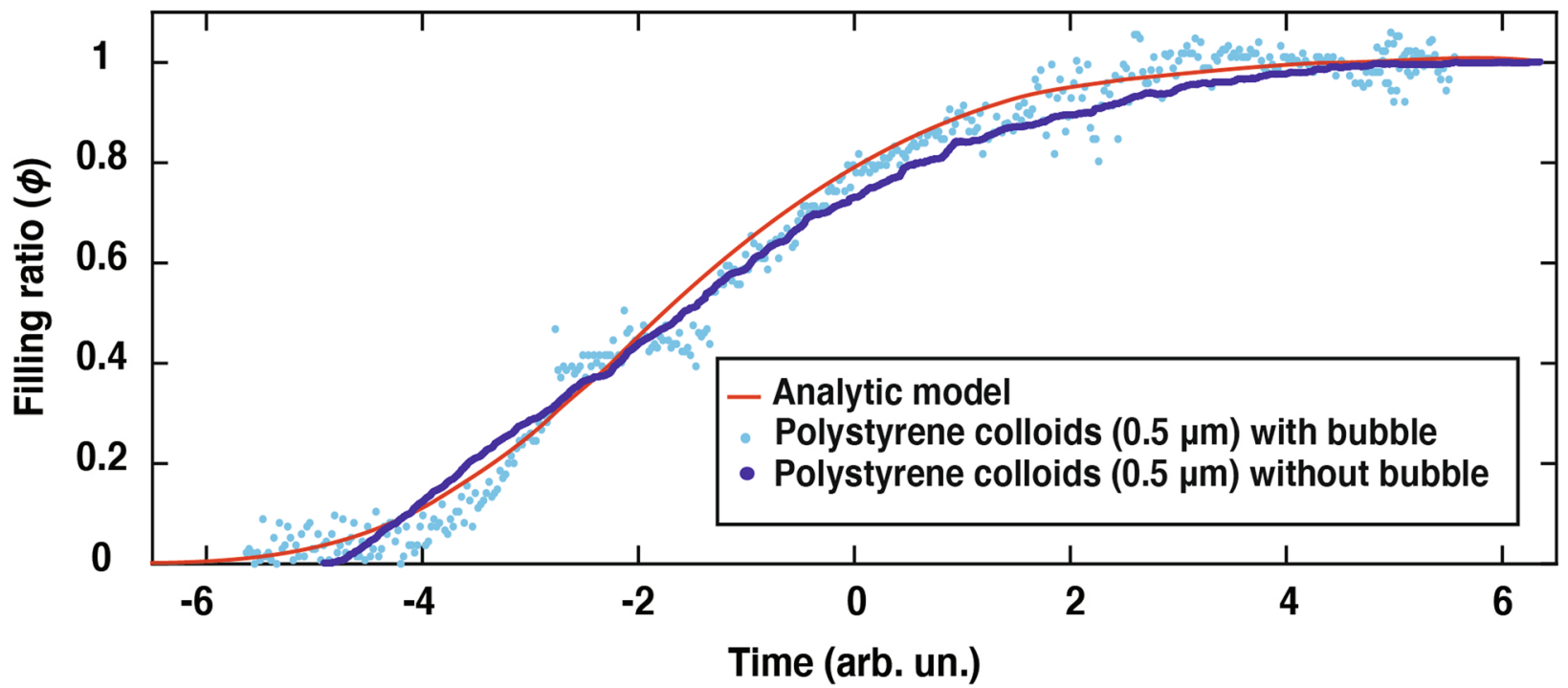

Extended Data Fig. 4 | Growth curves in the presence or absence of a cavitation bubble. Comparison of the growth curves of aggregates growing at and in the absence of a cavitation bubble. 


\section{NATURE PHYSICS}
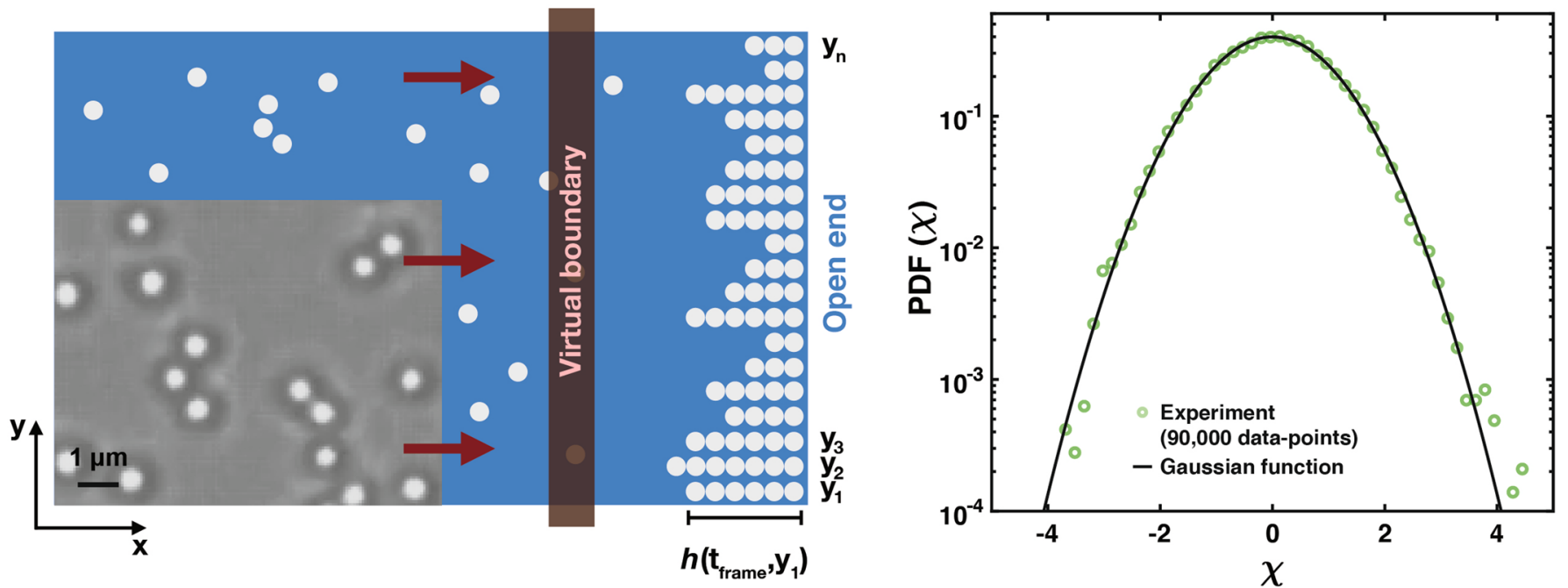

Extended Data Fig. 5 | Interface fluctuations of a virtual aggregate. Illustration and microscopy image (inset) showing the control experiment. Arrows denote the direction of the fluid flow towards the open end of sample. Particles are assumed to be collected passing the virtual boundary. Interface fluctuations were calculated using average width $\left(y_{n}\right)$ and height $\left(h\left(t_{\text {framer }} y_{n}\right)\right)$ of the growing aggregates. Semi-log scale plot showing experimentally obtained probability distribution function of the interface fluctuations (green dots). A ×100 objective was used for the imaging. 

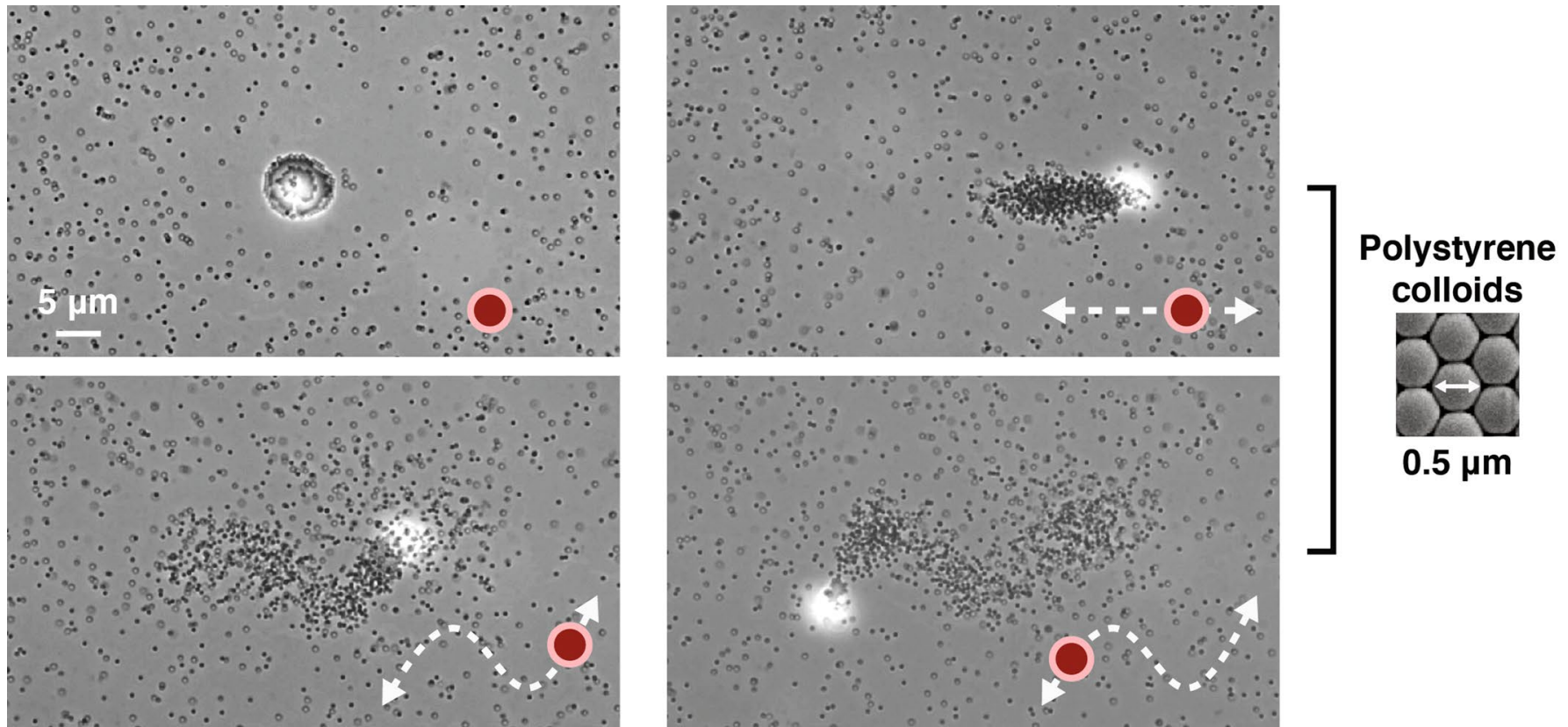

colloids

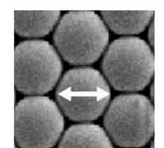

$0.5 \mu \mathrm{m}$

Extended Data Fig. 6 | Control over the aggregate movement. Time-lapse microscopy images showing an aggregate of 0.5- $\mu \mathrm{m}$ polystyrene colloids following the movement of laser beam to form a line (top), and a wave-like pattern (bottom). The bright white dot is the laser beam. The transmission of a small portion of the infrared beam is allowed from the visible lowpass filter (with relatively low attenuation to infrared) to show its exact position. Illustrated red dots show the movement of the beam. $\times 60$ objective was used for the imaging. . 


\section{NATURE PHYSICS}

ARTICLES

a

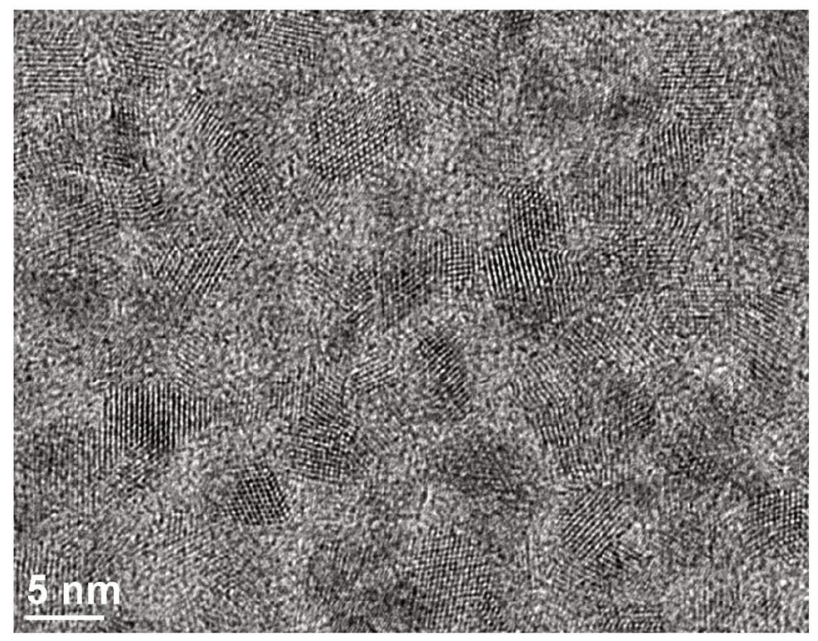

b

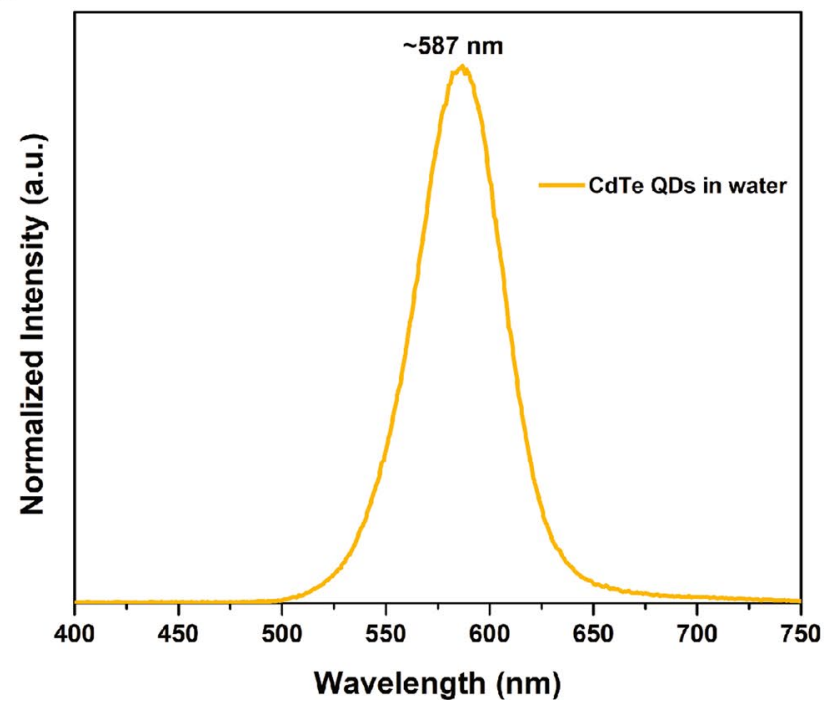

Extended Data Fig. 7 | CdTe characterization. a, Transmission electron microscopy image and (b) photoluminescence spectrum of the aqueous CdTe quantum dots. 
a
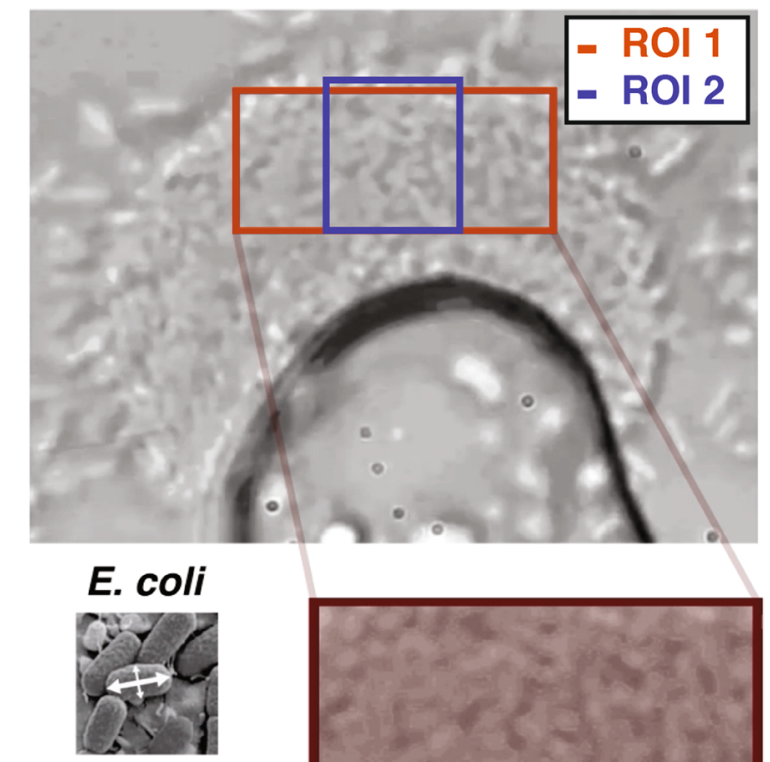

$\sim 1 \mu \mathrm{m} \times 2 \mu \mathrm{m}$

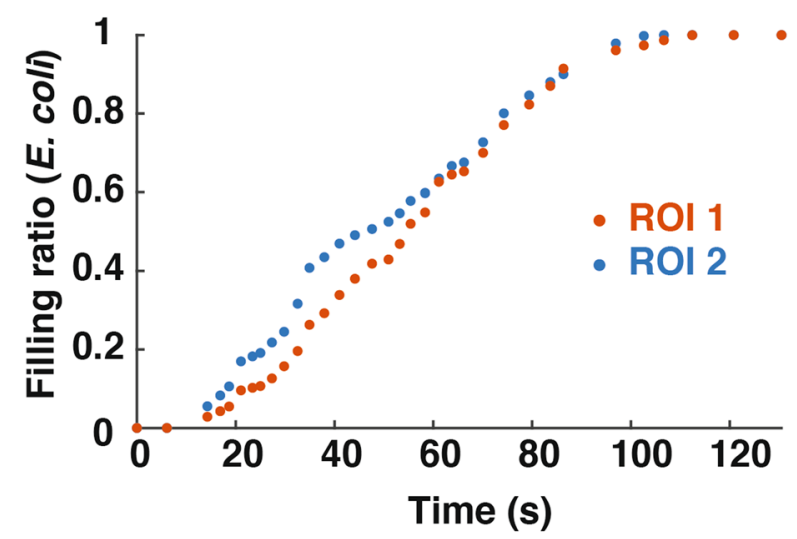

C

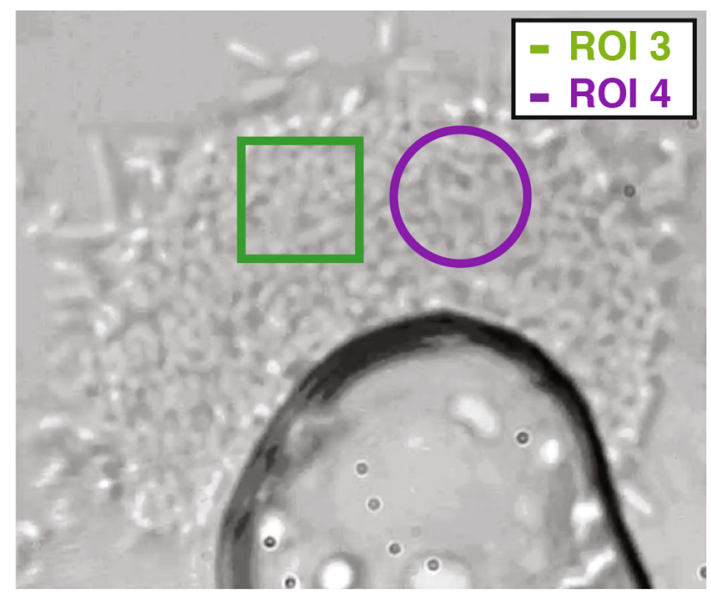

b
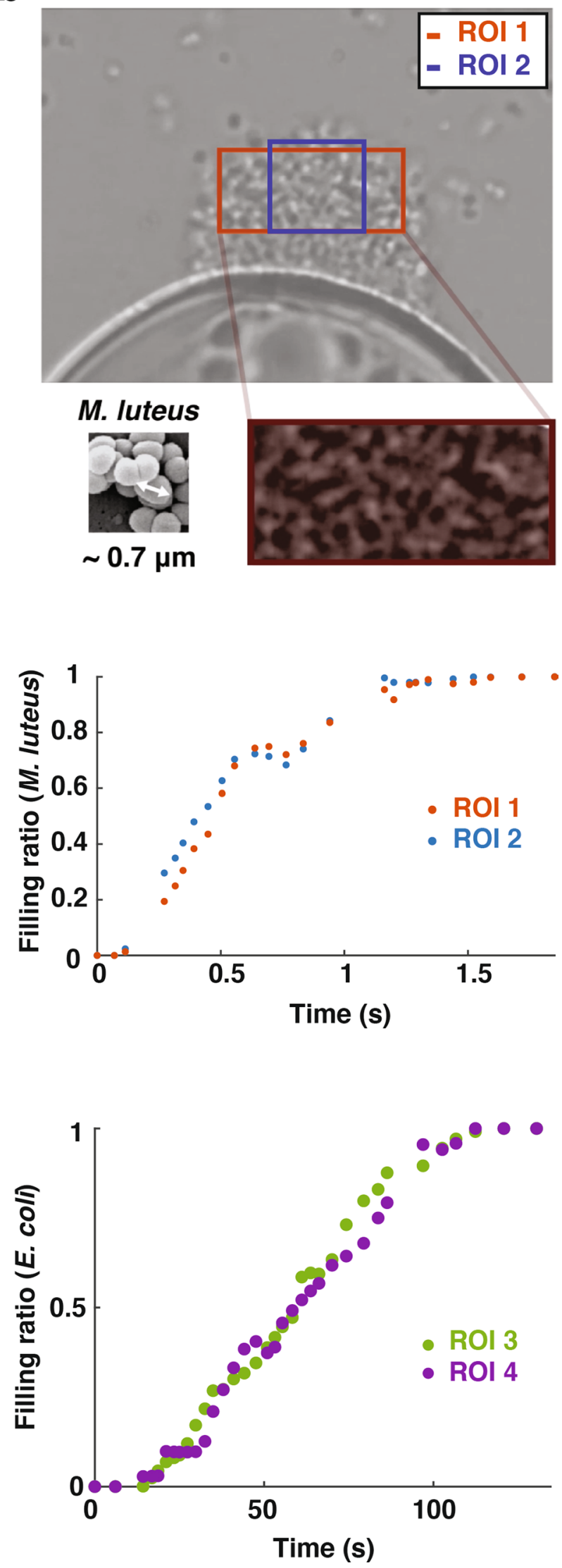

Extended Data Fig. 8 | Filling ratios for different region of interests. Filling ratio of different selected areas (region of interest: ROI) with E.coli and $M$. Luteus bacterial cells during their aggregation. Areas with different sizes (ROI 1, 2, and 3 of (a), (b), and (c)), shapes (a square, ROI 3, and a circle, ROI 4), and positions within the aggregate (c) do not affect the filling ratio. 


\section{NATURE PHYSICS}

a

$t_{0}$

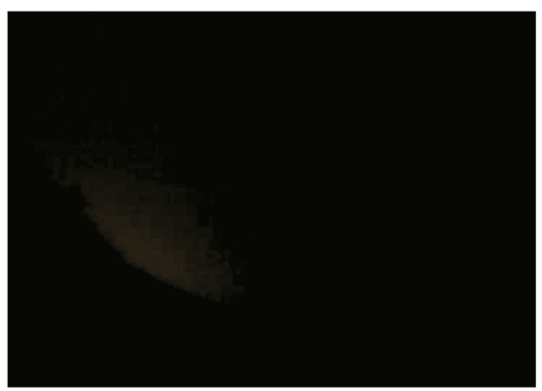

b

$$
t_{0}+40 s
$$

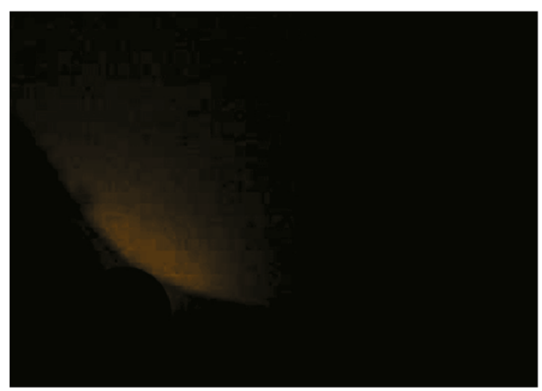

C

$$
t_{0}+80 s
$$
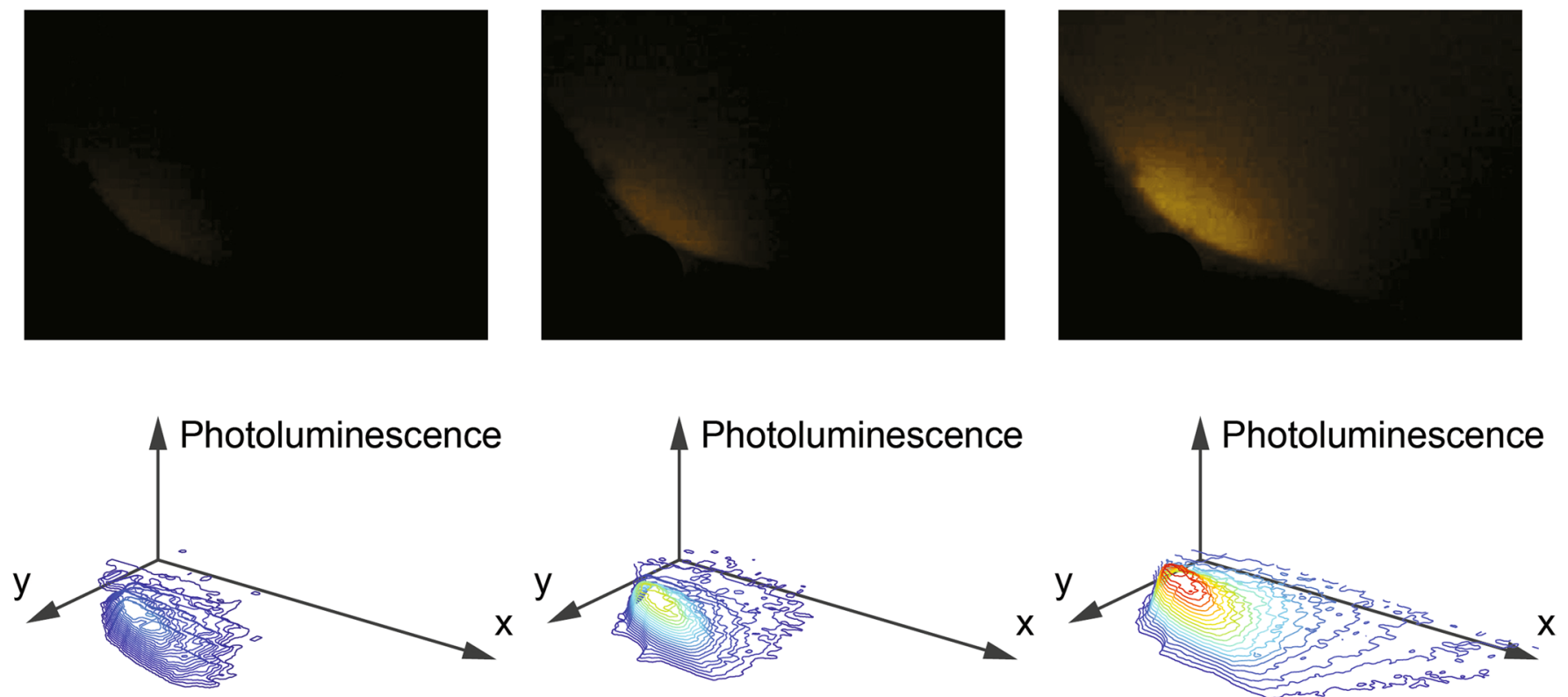

Extended Data Fig. 9 | Detection of CdTe photoluminescence signal. Time-lapse microscopy images (top) and plots (bottom) showing detection of photoluminescence signal emitted from growing $\mathrm{CdTe}$ quantum dot aggregate. A ×40 objective was used for the imaging. 


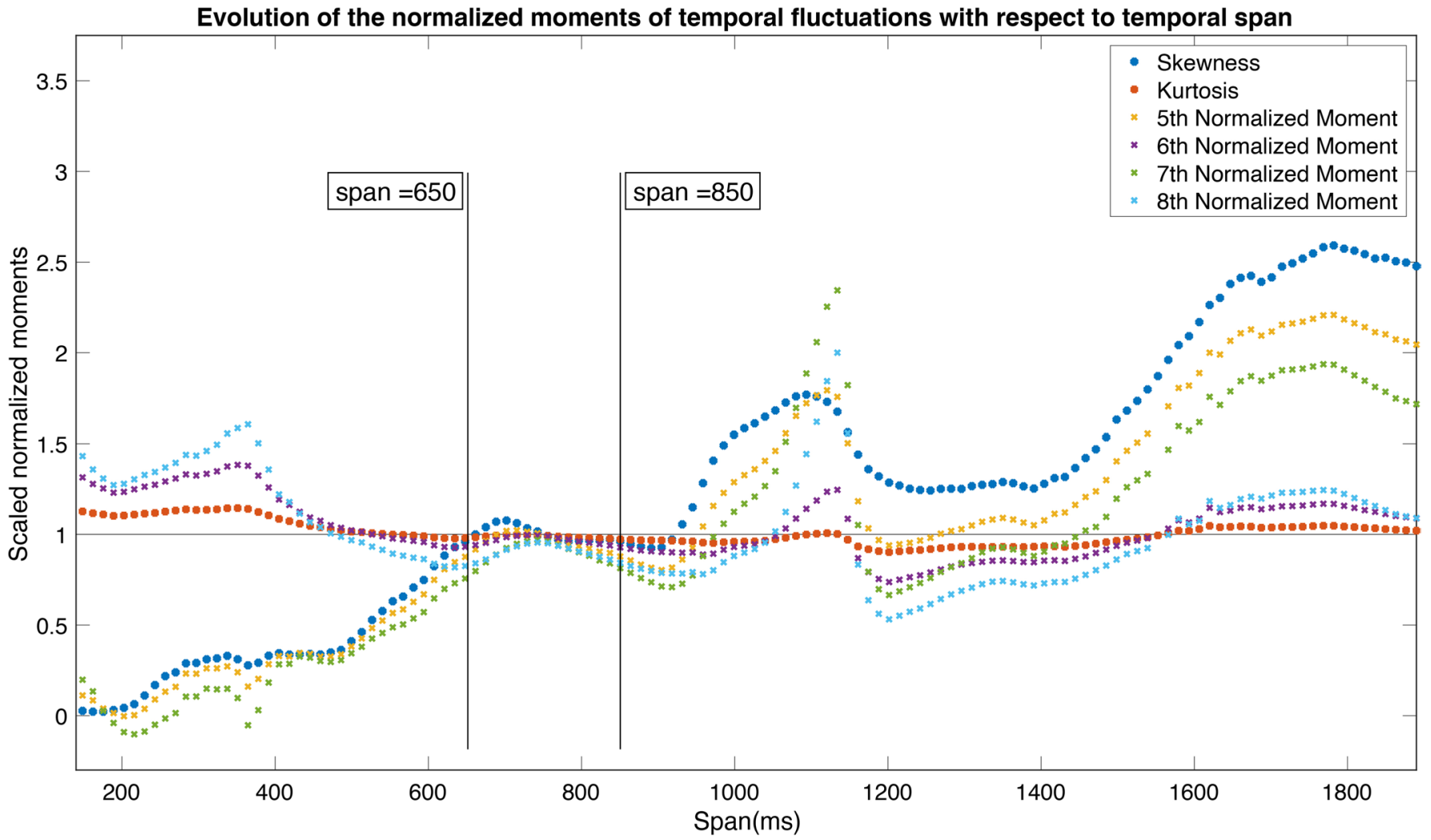

Extended Data Fig. 10 | Tracy-Widom GUE temporal span analysis. The evolution of the scaled normalized moments of temporal fluctuations with that of Tracy-Widom GUE with respect to temporal span. 


\section{natureresearch}

Corresponding author(s): Serim Ilday

Last updated by author(s): 24/02/20

\section{Reporting Summary}

Nature Research wishes to improve the reproducibility of the work that we publish. This form provides structure for consistency and transparency in reporting. For further information on Nature Research policies, see Authors \& Referees and the Editorial Policy Checklist.

\section{Statistics}

For all statistical analyses, confirm that the following items are present in the figure legend, table legend, main text, or Methods section.

n/a Confirmed

$\square$ \The exact sample size $(n)$ for each experimental group/condition, given as a discrete number and unit of measurement

$\square$ \A statement on whether measurements were taken from distinct samples or whether the same sample was measured repeatedly

$\triangle$ The statistical test(s) used AND whether they are one- or two-sided

$\triangle \square$ Only common tests should be described solely by name; describe more complex techniques in the Methods section.

\ $\square$ A description of all covariates tested

$\square$ \ A description of any assumptions or corrections, such as tests of normality and adjustment for multiple comparisons

$\square$ A full description of the statistical parameters including central tendency (e.g. means) or other basic estimates (e.g. regression coefficient)

$\triangle$ AND variation (e.g. standard deviation) or associated estimates of uncertainty (e.g. confidence intervals)

$\bigotimes$ For null hypothesis testing, the test statistic (e.g. $F, t, r$ ) with confidence intervals, effect sizes, degrees of freedom and $P$ value noted

$\triangle$ Give $P$ values as exact values whenever suitable.

Х $\square$ For Bayesian analysis, information on the choice of priors and Markov chain Monte Carlo settings

Х $\square$ For hierarchical and complex designs, identification of the appropriate level for tests and full reporting of outcomes

$\bigotimes \square$ Estimates of effect sizes (e.g. Cohen's $d$, Pearson's $r$ ), indicating how they were calculated

Our web collection on statistics for biologists contains articles on many of the points above.

\section{Software and code}

Policy information about availability of computer code

Data collection MATLAB

Data analysis MATLAB

For manuscripts utilizing custom algorithms or software that are central to the research but not yet described in published literature, software must be made available to editors/reviewers. We strongly encourage code deposition in a community repository (e.g. GitHub). See the Nature Research guidelines for submitting code \& software for further information.

\section{Data}

Policy information about availability of data

All manuscripts must include a data availability statement. This statement should provide the following information, where applicable:

- Accession codes, unique identifiers, or web links for publicly available datasets

- A list of figures that have associated raw data

A description of any restrictions on data availability

All data needed to evaluate the conclusions in the paper are present in the paper and/or the supplementary materials. Additional data, code, and materials related to this paper may be requested from the corresponding author.

\section{Field-specific reporting}

Please select the one below that is the best fit for your research. If you are not sure, read the appropriate sections before making your selection. \ Life sciences 


\section{Life sciences study design}

All studies must disclose on these points even when the disclosure is negative.

Sample size Details have been given in the main manuscript, methods section, and in the supplementary information document.

Data exclusions No data has been excluded.

Replication Details have been given in the main manuscript, methods section, and in the supplementary information document.

Randomization Not applicable.

Blinding Not applicable.

\section{Reporting for specific materials, systems and methods}

We require information from authors about some types of materials, experimental systems and methods used in many studies. Here, indicate whether each material, system or method listed is relevant to your study. If you are not sure if a list item applies to your research, read the appropriate section before selecting a response.

Materials \& experimental systems Methods

$\mathrm{n} / \mathrm{a}$ Involved in the study

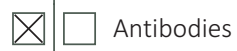

$\square$ Eukaryotic cell lines

$\square$ Palaeontology

Х $\square$ Animals and other organisms

\ $\square$ Human research participants

\ $\square$ Clinical data

\section{Eukaryotic cell lines}

Policy information about cell lines

Cell line source(s)

Human breast epithelial cell line - MCF-10A

Authentication

MCF-10A cells were authenticated by STR sequencing.

Mycoplasma contamination

MCF-10A cells were tested for mycoplasma contamination regularly using MycoAlert Mycoplasma Detection Kit (Lonza).

Commonly misidentified lines

(See ICLAC register) n/a Involved in the study

$\bigotimes \square$ ChIP-seq

\ $\square$ Flow cytometry

\ $\square$ MRI-based neuroimaging

No misidentified cell lines have been used during this study 